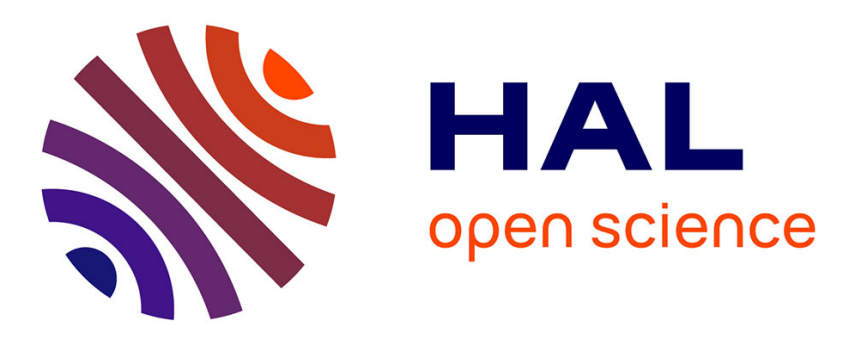

\title{
Assessing Climate Change Impact on the Spatial Dependence of Extreme Snow Depth Maxima in the French Alps
}

Gilles Nicolet, Nicolas Eckert, Samuel Morin, Juliette Blanchet

\section{- To cite this version:}

Gilles Nicolet, Nicolas Eckert, Samuel Morin, Juliette Blanchet. Assessing Climate Change Impact on the Spatial Dependence of Extreme Snow Depth Maxima in the French Alps. Water Resources Research, 2018, 54 (10), pp.7820-7840. 10.1029/2018wr022763 . hal-02404619

\author{
HAL Id: hal-02404619 \\ https://hal.science/hal-02404619
}

Submitted on 18 Nov 2021

HAL is a multi-disciplinary open access archive for the deposit and dissemination of scientific research documents, whether they are published or not. The documents may come from teaching and research institutions in France or abroad, or from public or private research centers.
L'archive ouverte pluridisciplinaire HAL, est destinée au dépôt et à la diffusion de documents scientifiques de niveau recherche, publiés ou non, émanant des établissements d'enseignement et de recherche français ou étrangers, des laboratoires publics ou privés. 


\section{Water Resources Research}

\section{RESEARCH ARTICLE \\ 10.1029/2018WR022763 \\ Assessing Climate Change Impact on the Spatial Dependence of Extreme Snow Depth Maxima in the French Alps}

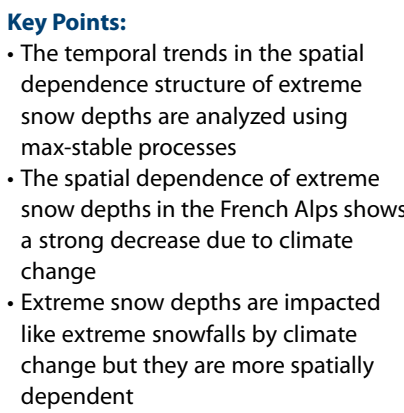

Correspondence to:

G. Nicolet,

gilles.nicolet@univ-grenoble-alpes.fr

Citation:

Nicolet, G., Eckert, N., Morin, S., \& Blanchet, J. (2018). Assessing climate change impact on the spatial dependence of extreme snow depth maxima in the French Alps. Water Resources Research, 54, 7820-7840. https://doi.org/10.1029/2018WR022763

Received 12 FEB 2018 Accepted 11 SEP 2018 Accepted article online 21 SEP 2018 Published online 16 OCT 2018

(92018. American Geophysical Union. All Rights Reserved.

\author{
Gilles Nicolet ${ }^{1,2,3}$ iD, Nicolas Eckert ${ }^{2}$ iD, Samuel Morin ${ }^{3}$, and Juliette Blanchet ${ }^{1}$ iD \\ ${ }^{1}$ Univ. Grenoble Alpes, Grenoble INP, CNRS, IRD, IGE, Grenoble, 38000, France, ${ }^{2}$ Université Grenoble Alpes, Irstea, UR ETGR, \\ St-Martin-d'Hères, France, ${ }^{3}$ Univ. Grenoble Alpes, Université de Toulouse, Météo-France, CNRS, CNRM, Centre d'Etudes de \\ la Neige, Grenoble, France
}

Abstract Modeling extreme snow depths in space is important for water storage, tourism industry, mountain ecosystems, collapse of buildings, and avalanche prevention. However, studies modeling the spatial dependence structure of extremes generally assume temporal stationarity which is clearly questionable in a climate change context. We model climatic trends within the spatial dependence structure of extremes, with application to a data set of snow depth winter maxima. From 82 stations spanning the 1970-2012 period in the French Alps, we infer a strong decrease in the range of spatial extremal dependence. This finding is related to a strong decrease in both the snow precipitation ratio and the winter cumulated snowfall, due to increasing temperatures. Hence, we demonstrate that the spatial dependence of extreme snow depths is impacted by climate change in a similar way as has been observed for extreme snowfalls. Furthermore, snow depths maxima are more spatially dependent than snowfalls. The space-time approach that we introduce may be very useful for assessing past and future evolutions under ongoing climate change in various hydrological quantities.

\section{Introduction}

Snow cover is an important source of water in mountainous regions, and extreme snow depths have thus a strong impact on water storage (Ehrler et al., 1997). Extreme snow depths further may have consequences on tourism industry (Koenig \& Abegg, 1997) and on mountain ecosystems (Keller et al., 2005; Wipf et al., 2009). Moreover, they also may cause collapse of buildings (Geis et al., 2011; Taylor, 1979) and extreme snow depth values are relevant for avalanche prevention (Schweizer et al., 2009). Hence, modeling extreme snow depths as precisely as possible is important for various environmental issues.

Snow depths show a strong spatial dependence in extremes, that is to say, close-enough locations are likely to experience concomitant extremes (Blanchet \& Davison, 2011), so that the spatial dependence structure of extremes is a valuable piece of information that is advantageous to model. This can be done through max-stable processes (de Haan, 1984), which are the spatial extension of univariate extreme value theory (Coles, 2001). Dombry et al. (2012) showed how to make predictions at ungauged locations by simulating max-stable processes conditionally on observations and applied this to precipitation and temperature data. Gaume et al. (2013) considered max-stable processes to built conditional return level maps of extreme snowfalls in the French Alps. Conditional simulations of max-stable processes were used by Oesting et al. (2017) to downscale precipitation data in southeastern France. Blanchet and Creutin (2017) studied the spatial nonstationarity of extreme rainfall coocurrence in the French Mediterranean region using max-stable processes. By considering temporal moving windows in a max-stable process framework, Blanchet et al. (2018) highlighted large differences in the temporal evolution of the cooccurrence of extreme precipitation in Senegal and in Central Sahel since 1950. Max-stable processes offer a suitable framework to deal with spatial extremes by modeling their spatial dependence structure together with the distribution of their amplitude. They have been widely applied within hydrology (Asadi et al., 2015; Reich \& Shaby, 2012; Shang et al., 2011; Thibaud et al., 2013; Westra \& Sisson, 2011) and especially to extreme snow depths in Switzerland (Blanchet \& Davison, 2011) and to extreme snowfalls and to avalanche slab depths in the French Alps (Gaume et al., 2012, 2013; Nicolet et al., 2016, 2017).

As pointed out in Beniston et al. (2018), the prediction of the future evolution of snow variables is particularly challenging due to the exacerbated response of the cryosphere to warming. The issue of temporal nonstation- 

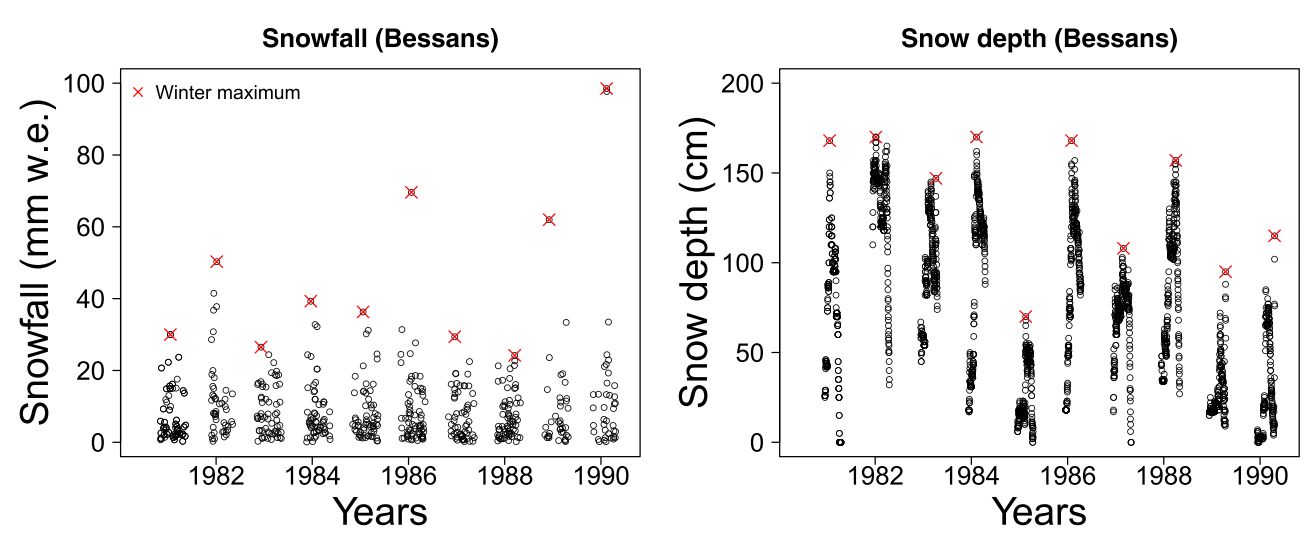

Figure 1. Daily snowfall (Nicolet et al., 2017) and snow depth (this study) series Bessans, in the French Alps, from winter 1981 to winter 1990. Red crosses indicate winter maxima.

arity in the modeling of extreme snow depths is therefore crucial, so as to realistically anticipate the evolution of the related risks under climate change and to assess more accurately risk metrics such as high quantiles or conditional tail expectation (under the condition of adapting these risk metrics to the nonstationary case). This is especially true for the modeling of the temporal nonstationarity within the dependence structure, for which the methodological background currently available remains particularly weak. Indeed, only few studies have to date applied max-stable processes with spatiotemporal dependence structures to case studies (Huser \& Davison, 2014; Raillard, 2011; Steinkohl, 2013) and all of them have considered the short-range temporal dependence of extremes rather than temporal nonstationarity in the spatial dependence at decadal time scale. Although Blanchet et al. (2018) investigated the temporal evolution of spatial dependence of extreme precipitation in West Africa during the last decades, they did not explicitly model temporal trends within the spatial dependence structure but fitted stationary max-stable processes on moving-time windows. Nicolet et al. (2016) used a data-based approach (without explicitly fitting a complete max-stable model) to highlight a negative temporal trend in the spatial dependence of extreme snowfalls in the French Alps. Although detection of temporal trends in the dependence structure was thus possible, this did not allow modeling the temporal evolution and is thus not usable to make predictions.

Nicolet et al., 2016's (2016) results concern extreme snowfalls. This does not permit to draw conclusions regarding snow depths, because these two variables, although connected, are actually very different. Indeed, as shown in Figure 1, snowfall is an instantaneous variable while snow depth is cumulative and exhibits a much smoother temporal evolution. Snow depths are more than a simple sum of snowfalls because they involve many other factors (snow compaction, snowmelt, etc. ), which induce large differences in their extremes. For instance, as shown in Figure 1, their most extreme values do not necessarily occur during the same winters. Moreover, due to the cumulative effects involved in the formation of the snowpack, the spatial dependence between extremes appears stronger for snow depths than for snowfalls (Gaume et al., 2013). Yet this has never been demonstrated so far for snowfalls and snow depths data from the same region.

On this basis, the objective of this paper is to propose a first approach to account for long-term climate nonstationarity in the spatial dependence structure of extreme snow depths using max-stable processes. This approach is used to infer the temporal evolution of the spatial dependence in extreme snow depths under climate change. To this aim, we process a data set of 82 snow depth winter maxima series in the French Alps. Specifically, we fit different Brown-Resnick (Kabluchko et al., 2009) processes with temporal trends in the spatial structure of dependence. For each model, the temporal trend is conveyed by different local and synoptic covariates and a rigorous procedure is used to highlight the most relevant ones. This allows us to highlight the impact of climate change on the spatial dependence structure of extreme snow depths. The comparison with previous results on extreme snowfalls over the same area (French Alps) and during the same time scale (last decades) allows us to conveniently compare extreme snow depths and extreme snowfalls and to identify similarities and differences concerning their response to climate change. 


\section{Data Set}

\subsection{Snow Depths}

Our data set is composed of snow depth winter maxima with a winter period defined from 15 November to 15 May and extracted from three databases:

1. twice-daily manual measurements (dedicated snow manual observing network) from 1970 to 2013 (17 stations);

2. daily automatic measurements (Nivôse stations) from 1980 to 2013 (seven stations);

3. weekly manual measurements (dedicated snow manual observing network) from 1983 to 2013 (58 stations).

This provides a data set of 82 stations in the French Alps (Figure 2a), from which the annual winter maxima are extracted. Although there are many missing data before 1983 (Figure 2b), the 18 stations with more than 30 winter maxima (in red in Figure 2a) are relatively uniformly distributed over the study area. In addition, the coverage of station elevation is very appropriate to address mountainous conditions, with many stations above 2,000 m (Figure 2c).

\subsection{Covariates}

We consider time, reanalyses of winter cumulated snowfall, winter snow precipitation ratio and temperature (daily minimum, maximum, and mean; Durand, Laternser, et al., 2009), NAO (North Atlantic Oscillation; Jones et al., 1997; Osborn, 2006), and AMO (Atlantic Multidecadal Oscillation ; Enfield et al., 2001; Kaplan et al., 1998). Variables stemming from reanalyses (cumulated snowfall, snow precipitation ratio, minimum temperature, mean temperature, and maximum temperature) are considered at two elevation levels: 1,800 m and 2,400 m. For each covariate, we retain a single value for each winter and for the entire French Alps. Details are given in Appendix A.

Since we are interested in modeling climate effects in the spatial dependence structure of extreme snow depths, we remove the annual variability in the covariates by considering time moving averages. In this article, we consider 17-year moving averages (Figure 3). The value $c_{k}(n)$ of the covariate $c_{k}$ for winter $n$ is then the average of the raw values of this covariate from the winter $n-8$ to the winter $n+8$. For all the covariates, we have at our disposal all the data before 1970 required to compute the first moving averages without missing data. However, for the most recent time windows the average is computed on restricted windows, using data up to 2012.

In the considered period, we observe decrease in snow precipitation ratio at 1,800 $\mathrm{m}$ and cumulated snowfall and increase in maximum temperature, mean temperature, and AMO. The period of strongest decrease in cumulated snowfall coincides with that of snow precipitation ratio at 1,800 $\mathrm{m}$ and 2,400 $\mathrm{m}$, extending from 1982 to 1995 . Temperature and AMO mainly increase during this period. The period of strongest increase is larger for AMO (from 1978 to 2002) and starts earlier (around 1977) for maximum and mean temperature. NAO increases before 1985 and decreases after 1994. At 2,400 m, the snow precipitation ratio is close to 1 during the entire study period, which means that, at this elevation, most of the precipitation falls as snow from 15 November to 15 May regardless of the year.

The correlation table (Table 1) shows that many of our covariates are strongly correlated. Although considering moving averages makes these correlations higher than when using raw annual values, the strength of these correlations is mainly due to the strong physical connections between these variables. There are 37 pairs of covariates showing a correlation lower than 0.8 in absolute value. Only these pairs are considered for the nonstationary models with several covariates in order to avoid inference problems related to redundant information.

For computation efficiency, each covariate $c_{k}$ is zero centered as follows:

$$
C_{k}(n)=\frac{c_{k}(n)-\overline{c_{k}}}{\operatorname{std}\left(c_{k}\right)}
$$

with $\overline{c_{k}}$ and $\operatorname{std}\left(c_{k}\right)$ the mean and the standard deviation of $\left(c_{k}(1), \ldots, c_{k}(N)\right)$, respectively.

\section{Method}

3.1. Brown-Resnick Max-Stable Process

3.1.1. Definition of a Max-Stable Process

Let $\chi$ be the French Alps and $X_{n, i}(x)$ the daily snow depths in location $x \in \chi$ during the day $i$ of winter $n$ ( $1 \leq i \leq d_{0}$ with $d_{0}$ the number of days in winter). For each winter $n$ and for each location $x$, we define $Z_{n}(x)$ as 
(a)

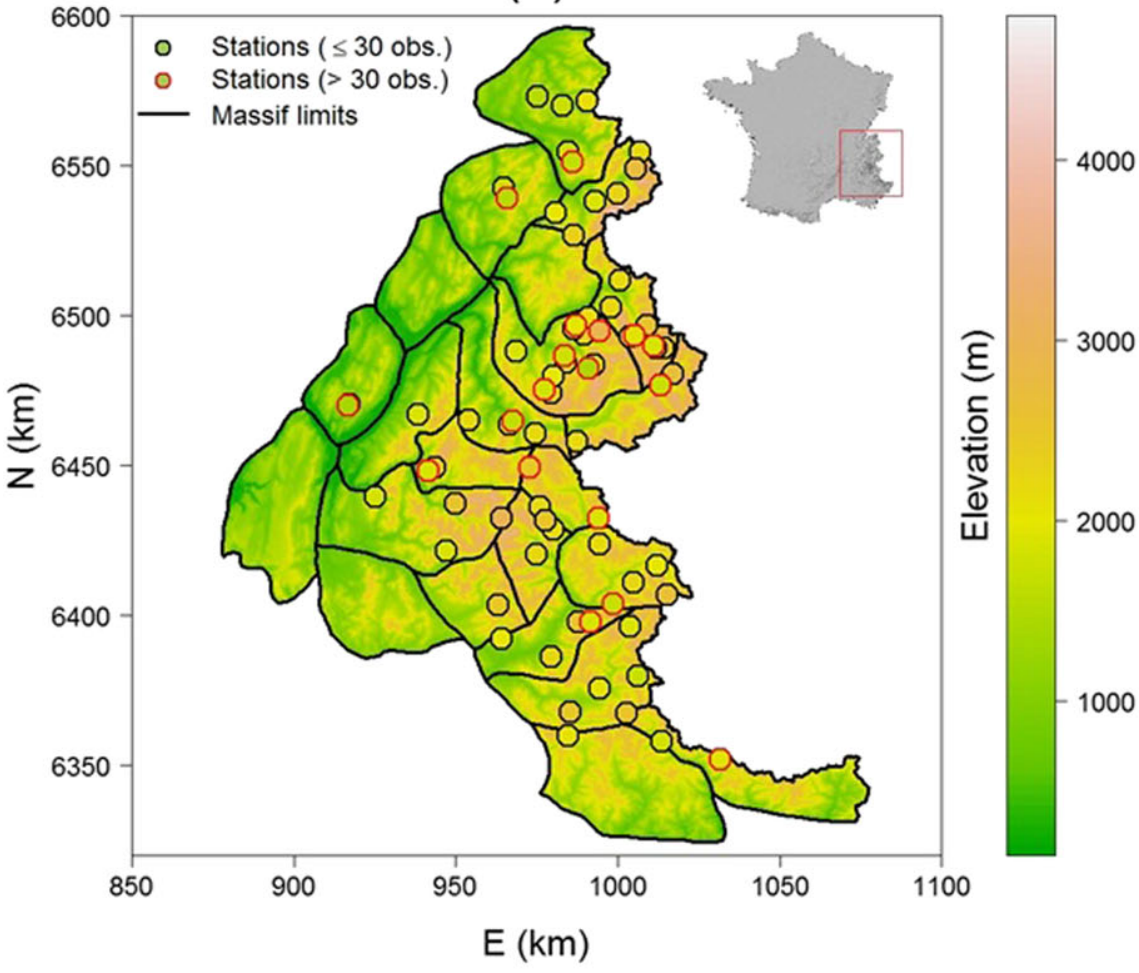

(b)

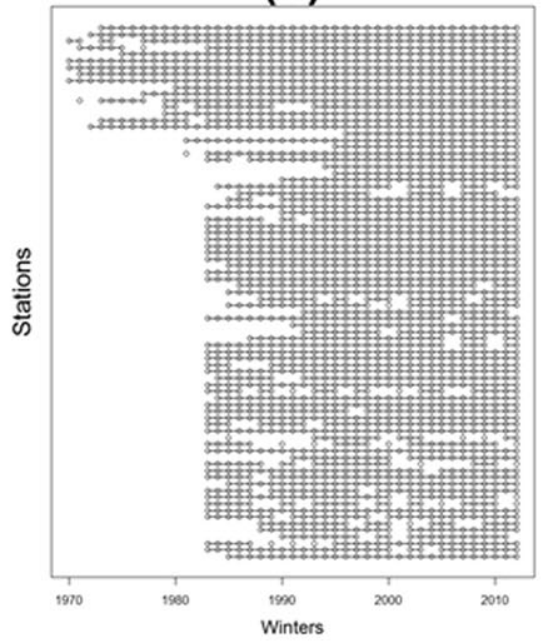

(c)

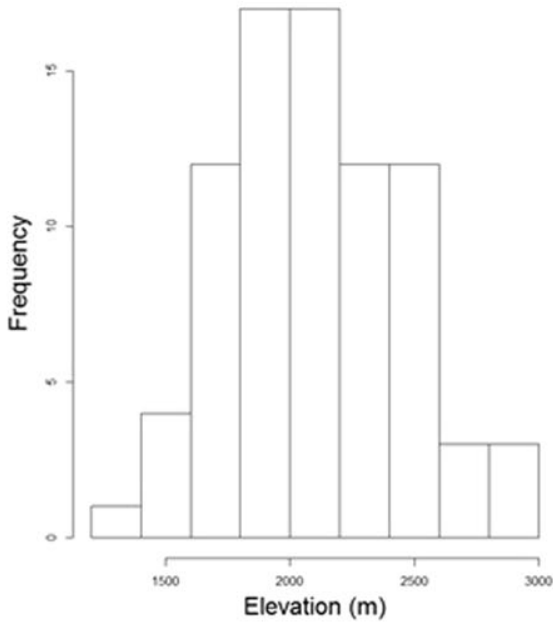

Figure 2. Snow depth data set (section 2.1). (a) Study area in the southeast of France, where the 23 massifs of the French Alps are located. Lines denote massif limits, and dots denote the positions of the stations. The stations having more than 30 annual maxima are indicated in red. (b) Data availability for each station. Each line represents one station, and each point means that the winter maxima is available for that station. (c) Histogram of station elevation.

winter maximum snow depth $Z_{n}(x)=\max _{1 \leq i \leq d_{0}} X_{n, i}(x)$. For instance, Figure 4 represents $Z_{n}(x)$ (standardized at each location in order to have the same distribution) for the two years 1983 and 2012 (i.e., the first and last year with enough data to have a convenient spatial coverage of the French Alps).

We consider these $Z_{n}(x)$ as the realizations of a spatial process $\{Z(x)\}_{x \in \chi}$. One can observe in Figure 4 that the largest maxima are likely to occur together with other large maxima in the closest locations. Actually, the closer the locations, the larger the probability to observe large maxima in these locations during the same winter. In this paper, we focus on this spatial dependence of snow depth maxima. According to Leadbetter (1983), 

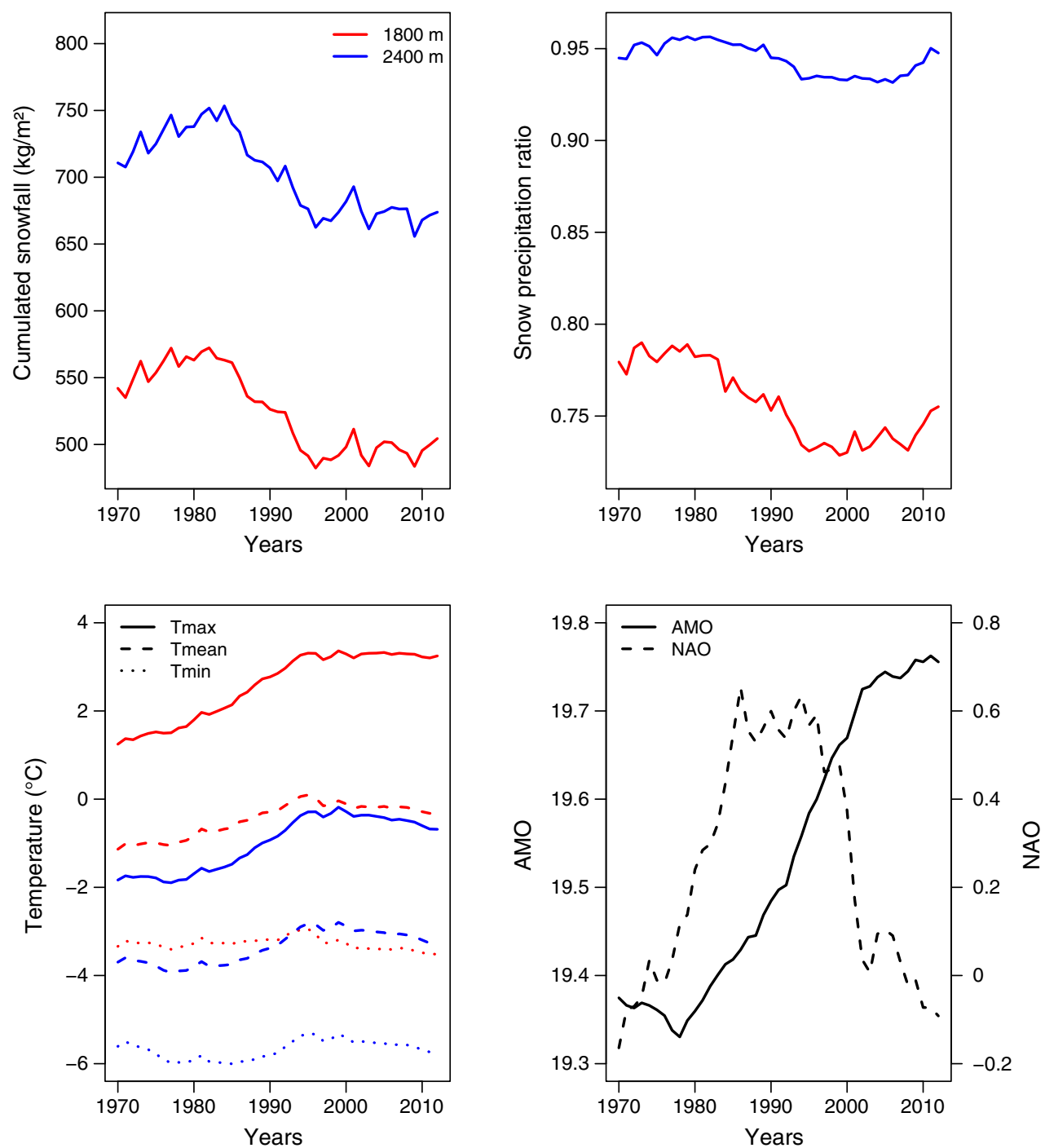

Figure 3. Covariates (section 2.2). The 17-year moving averages of the considered covariates: winter cumulated snowfall, snow precipitation ratio, and temperature (daily maximum, mean, and minimum) in the French Alps, AMO, and NAO indexes. The elevation at which the covariate (except AMO and NAO indexes) is considered is indicated by red $(1,800 \mathrm{~m})$ or blue $(2,400 \mathrm{~m})$ lines.

which allows us to consider maxima of daily snow depths despite their temporal dependence, and to spatial extreme value theory (Cooley et al., 2012; Davison et al., 2012), we know that the process $\{Z(x)\}_{x \in \chi}$ of winter maximum snow depths in the French Alps can be advantageously modeled as a max-stable process (de Haan, 1984). At each location $x \in \chi, Z(x)$ follows a generalized extreme value (GEV) $(\mu(x), \sigma(x), \xi(x))$ distribution. In this article, we do not investigate the issue of the distribution of $Z(x)$ but focus on the spatial dependence of $\{Z(x)\}_{x \in X}$ (i.e., the dependence between $Z\left(x_{1}\right)$ and $Z\left(x_{2}\right)$ with $x_{1}$ and $x_{2}$ two locations). That is why, for more convenience, the distribution of $Z(x)$ at each location $x$ can be transformed without loss of generality into unit Fréchet distribution (i.e., $\mathrm{GEV}(1,1,1)$ ) through the transformation

$$
Z(x) \mapsto \frac{-1}{\log [F\{Z(x) ; \mu(x), \sigma(x), \xi(x)\}]}
$$

with $F\{. ; \mu(x), \sigma(x), \xi(x)\}$ the cumulated distribution function of the $\operatorname{GEV}(\mu(x), \sigma(x), \xi(x))$ distribution.

\subsubsection{The Brown-Resnick Process}

Kabluchko et al. (2009) generalized the max-stable process previously introduced in Brown and Resnick (1977). By using this process to model the process $\{Z(x)\}_{x \in x}$ of winter maxima, the bivariate distribution of the winter 
Table 1

Covariates (Section 2.2); Correlation Table for the 17-Year Moving Averages of Covariates

\begin{tabular}{|c|c|c|c|c|c|c|c|c|c|c|c|c|}
\hline & NAO & AMO & $\mathrm{T}_{\mathrm{min}}^{1800}$ & $\mathrm{~T}_{\text {mean }}^{1800}$ & $\mathrm{~T}_{\max }^{1800}$ & $\mathrm{~T}_{\min }^{2400}$ & $\mathrm{~T}_{\text {mean }}^{2400}$ & $\mathrm{~T}_{\max }^{2400}$ & $\mathrm{SF}^{1800}$ & $\mathrm{SF}^{2400}$ & $\mathrm{SPR}^{1800}$ & $\mathrm{SPR}^{2400}$ \\
\hline Time & 0.01 & 0.97 & -0.32 & 0.84 & 0.94 & 0.46 & 0.80 & 0.89 & -0.84 & -0.82 & -0.85 & -0.69 \\
\hline NAO & 1 & -0.13 & 0.75 & 0.46 & 0.28 & -0.01 & 0.21 & 0.25 & -0.05 & 0.07 & -0.26 & -0.07 \\
\hline AMO & - & 1 & -0.36 & 0.80 & 0.91 & 0.60 & 0.85 & 0.90 & -0.90 & -0.89 & -0.87 & -0.79 \\
\hline $\mathrm{T}_{\min }^{1800}$ & - & - & 1 & 0.22 & -0.01 & 0.25 & 0.14 & 0.05 & 0.04 & 0.14 & -0.07 & -0.05 \\
\hline $\mathrm{T}_{\text {mean }}^{1800}$ & - & - & - & 1 & 0.97 & 0.62 & 0.92 & 0.97 & -0.86 & -0.78 & -0.94 & -0.78 \\
\hline $\mathrm{T}_{\max }^{1800}$ & - & - & - & - & 1 & 0.59 & 0.92 & 0.98 & -0.90 & -0.84 & -0.95 & -0.79 \\
\hline $\mathrm{T}_{\min }^{2400}$ & - & - & - & - & - & 1 & 0.86 & 0.73 & -0.80 & -0.79 & -0.73 & -0.87 \\
\hline $\mathrm{T}_{\text {mean }}^{2400}$ & - & - & - & - & - & - & 1 & 0.98 & -0.95 & -0.91 & -0.96 & -0.93 \\
\hline $\mathrm{T}_{\max }^{2400}$ & - & - & 一 & - & - & - & - & 1 & -0.94 & -0.89 & -0.97 & -0.88 \\
\hline $\mathrm{SF}^{1800}$ & - & - & - & - & - & - & - & - & 1 & 0.99 & 0.94 & 0.90 \\
\hline $\mathrm{SF}^{2400}$ & - & - & - & - & - & - & - & - & - & 1 & 0.88 & 0.87 \\
\hline $\mathrm{SPR}^{1800}$ & - & - & 一 & - & - & - & - & - & - & - & 1 & 0.91 \\
\hline
\end{tabular}

Note.Time, NAO, and AMO indexes, minimum temperature at $1,800 \mathrm{~m}\left(\mathrm{~T}_{\mathrm{min}}^{1800}\right)$, mean temperature at $1,800 \mathrm{~m}\left(\mathrm{~T}_{\mathrm{mean}}^{1800}\right)$, maximum temperature at $1,800 \mathrm{~m}\left(\mathrm{~T}_{\mathrm{max}}^{1800}\right)$, minimum temperature at $2,400 \mathrm{~m}\left(\mathrm{~T}_{\mathrm{min}}^{2400}\right)$, mean temperature at $2,400 \mathrm{~m}\left(\mathrm{~T}_{\text {mean }}^{2400}\right)$, maximum temperature at $2,400 \mathrm{~m}\left(\mathrm{~T}_{\max }^{2400}\right)$, cumulated snowfall at $1,800 \mathrm{~m}\left(\mathrm{SF} \mathrm{F}^{1800}\right)$, cumulated snowfall at $2,400 \mathrm{~m}\left(\mathrm{SF}^{2400}\right)$, snow precipitation ratio at $1,800 \mathrm{~m}\left(\mathrm{SPR}^{1800}\right)$, snow precipitation ratio at $2400 \mathrm{~m}\left(\mathrm{SPR}^{2400}\right)$. Correlations which are larger than 0.8 in absolute value are in bold.

maxima $Z\left(x_{1}\right)$ and $Z\left(x_{2}\right)$ at two locations $x_{1}$ and $x_{2}$ is given by

$$
\mathcal{P}\left(Z\left(x_{1}\right)<z_{1}, Z\left(x_{2}\right)<z_{2}\right)=\exp \left\{-\frac{1}{z_{1}} \Phi\left(\frac{a}{2}+\frac{1}{a} \log \frac{z_{2}}{z_{1}}\right)-\frac{1}{z_{2}} \Phi\left(\frac{a}{2}+\frac{1}{a} \log \frac{z_{1}}{z_{2}}\right)\right\}
$$

with $\Phi$ the distribution function of the standard normal distribution and $a=\sqrt{2 \gamma(h)}$ with $h$ the distance between $x_{1}$ and $x_{2}$. In this paper, we use a power semivariogram $\gamma(h)=(h / \lambda)^{\kappa}$ with $\lambda$ and $\kappa$ its scale and shape parameters, respectively.

\subsubsection{Extremal Coefficient and Brown-Resnick Extremal Function}

To assess the extremal dependence between two unit Fréchet random variables $Z_{1}$ and $Z_{2}$, one can use the extremal coefficient $\theta$ (Naveau et al., 2009; Schlather \& Tawn, 2003) defined by

$$
\mathcal{P}\left(Z_{1}<z, Z_{2}<z\right)=\exp \left\{\frac{-\theta}{z}\right\}=\mathcal{P}\left\{Z_{1}<z\right\}^{\theta}, \quad z>0
$$
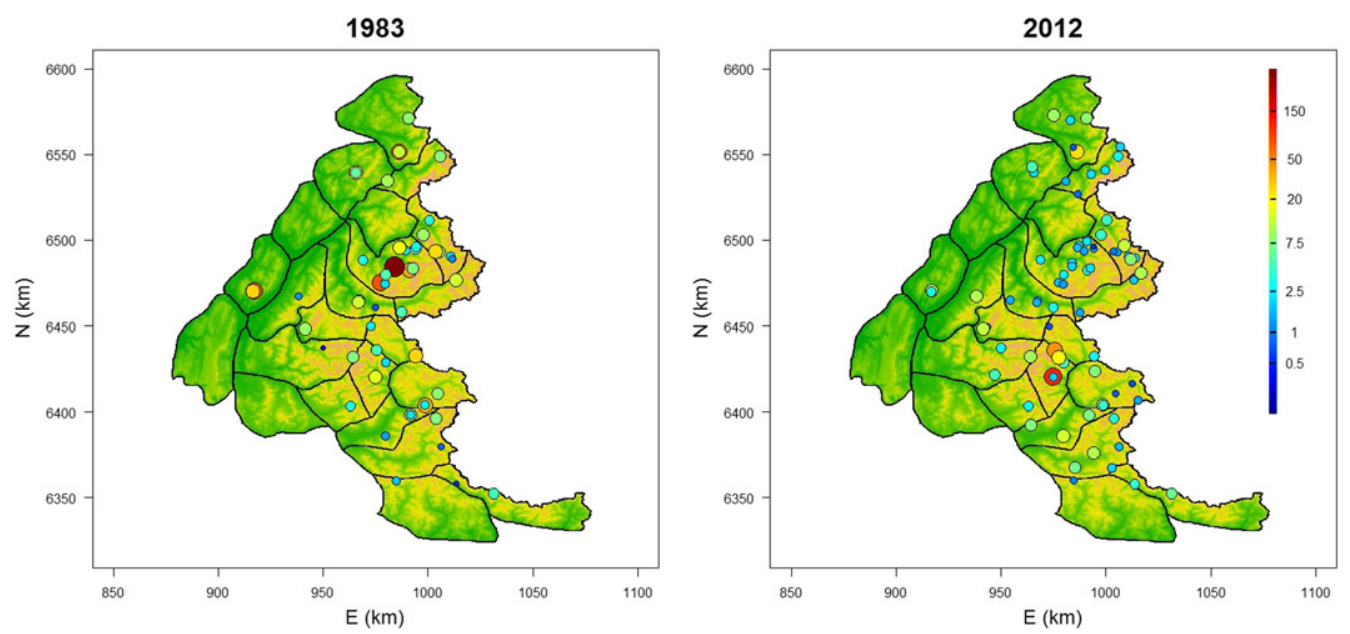

Figure 4. Snow depth maxima for the winters 1983 and 2012. These maxima are standardized into unit Fréchet at each location. The magnitude of the maxima is denoted by both the color code and the radius of the circles. The spatial clustering of high/low values shows the spatial structure of the considered process. 
The extremal coefficient ranges between 1 (complete dependence) and 2 (independence). The property

$$
\lim _{z \rightarrow \infty} \mathcal{P}\left(Z_{2}>z \mid Z_{1}>z\right)=2-\theta
$$

holds and means that the probability of observing extreme values of $Z_{2}$ when $Z_{1}$ takes extreme values is close to 0 when $\theta$ is near 2 and close to 1 when $\theta$ is near 1 .

If $Z_{1}=Z\left(x_{1}\right)$ and $Z_{2}=Z\left(x_{2}\right)$ with $Z$ a max-stable process and $x_{1}$ and $x_{2}$ two positions, we have theoretical expressions for $\theta$ (Ribatet, 2013) as functions $\theta(h)$ of the distance $h=\left|x_{2}-x_{1}\right|$. The expression $\theta(h)$ represents the strength of the dependence as a function of distance, and is therefore termed the extremal function. Specifically, the Brown-Resnick extremal function is given by

$$
\theta(h)=2 \Phi\left(\frac{\sqrt{2(h / \lambda)^{\kappa}}}{2}\right)
$$

with a power semivariogram.

\subsubsection{Range of Extremal Dependence}

As in Nicolet et al. (2016), we define the range of extremal dependence as the distance $h_{0}$ such that $\theta\left(h_{0}\right)=1.9$. The range denotes the distance above which snow depth maxima become weakly dependent in extremes, that is, close to independence in practice. The stronger the extremal dependence at large distances, the larger the range. Inverting (6) gives the following expression of the range:

$$
h_{0}(\lambda, \kappa)=\lambda\left[2\left\{\Phi^{-1}\left(\frac{1.9}{2}\right)\right\}^{2}\right]^{1 / \kappa} \text {. }
$$

\subsubsection{Modeling Anisotropy in the Spatial Dependence Structure}

Extremal dependence is generally spatially anisotropic due to the impact of different factors, such as the relief, atmospheric fluxes, etc. Thus, the modeling of extremal dependence between two locations as function of the Euclidean distance only may be too simplistic. In order to account for spatial anisotropy, we use a geometric transformation. As in Blanchet and Davison (2011) and in Nicolet et al. $(2016,2017)$, the vector of the coordinates $x$ in $\mathcal{R}^{3}$ is replaced with $x^{\prime}=V x$ such that

$$
V=\left(\begin{array}{ccc}
\cos \psi & -\sin \psi & 0 \\
R_{1}^{-1} \sin \psi & R_{1}^{-1} \cos \psi & 0 \\
0 & 0 & R_{2}
\end{array}\right)
$$

with $\psi$ the anisotropy angle, $R_{1}>1$ the anisotropy ratio and $R_{2}>0$ the weight parameter for elevation. The angle $\psi$ is the direction of strongest dependence. The parameter $R_{1}$ controls the ratio between the direction of strongest dependence and the orthogonal direction in the 2-D plane. The parameter $R_{2}$ is expected to be widely greater than 1 as a consequence of the strong influence of elevation on snow quantities. The distance computed after this transformation is referred to as the modified 3-D distance.

\subsubsection{Modeling Trend in the Spatial Dependence Structure}

Temporal trends related to changes in climate drivers within the spatial dependence structure are modeled by linear combinations of covariates for the two parameters $\lambda$ and $\kappa$ of the dependence structure. That is to say

$$
\left\{\begin{array}{l}
\lambda(n)=\lambda_{0}+\Sigma_{m=1}^{M_{1}} C_{\lambda, m}(n) \lambda_{m} \\
\kappa(n)=\kappa_{0}+\Sigma_{m=1}^{M_{2}} C_{\kappa, m}(n) \kappa_{m} .
\end{array}\right.
$$

with $C_{\lambda}(n)=\left\{C_{\lambda, k}(n)\right\}_{k \in\left\{1, \ldots, M_{1}\right\}}$ and $C_{\kappa}(n)=\left\{C_{\kappa, k}(n)\right\}_{k \in\left\{1, \ldots, M_{2}\right\}}$ as the two sets of time-dependent standardized variables ( $n$ indicates the winter). Allowing different covariates for $\lambda$ and $\kappa$ (i.e., $C_{\lambda}$ different from $C_{\kappa}$ ) allows for more flexibility.

\subsection{Inference and Model Selection}

3.2.1. Inference

In this study, our focus is in the spatial dependence of snow depth maxima and not in the marginal distribution. In other words, the marginal distributions are previously estimated pointwise by maximum likelihood, in which case studying the spatial distribution of maxima is equivalent to studying that of normalized maxima with the same margins. For spatial extremes, it turns out to be convenient to consider normalization to unit Fréchet. 
Due to computational issues, the full log-likelihood of max-stable processes is usually intractable (Castruccio et al., 2016). To estimate the parameters of a Brown-Resnick max-stable process, we can maximize the pairwise composite log-likelihood (Padoan et al., 2010)

$$
I(\beta)=\sum_{n=1}^{N} \sum_{i=1}^{K-1} \sum_{j=i+1}^{K} \log f\left(z_{n, i}, z_{n, j} ; C_{\lambda}(n), C_{\kappa}(n), \beta\right)
$$

with $K$ the number of stations, $N$ the number of years, $z_{n, i}$ the maxima at location $i$ for winter $n, f$ the bivariate density function associated to the Brown-Resnick distribution (3), and $C_{\lambda}(n)=\left\{C_{\lambda, k}(n)\right\}_{k \in\left\{1, \ldots, M_{1}\right\}}$ and $C_{\kappa}(n)=\left\{C_{\kappa, k}(n)\right\}_{k \in\left\{1, \ldots, M_{2}\right\}}$ the considered time-dependent standardized covariates, if any, and $\beta$ the vector of parameters of the bivariate distribution. In the case where the anisotropy parameters are not estimated, the vector $\beta$ consists of the $M_{1}+1$ parameters for $\lambda(n)$ and the $M_{2}+1$ parameters for $\kappa(n)$. Thus, a total of $M_{1}+M_{2}+2$ parameters have to be estimated (thus, two in total when no covariates are considered, as in the stationary and moving time window cases of sections 4.2 and 4.3). In the case where the three parameters $\psi$, $R_{1}$, and $R_{2}$ for anisotropy (which are supposed to be time independent) are estimated, $M_{1}+M_{2}+5$ parameters have to be estimated.

\subsubsection{Composite Likelihood Information Criterion}

The classical inference criterion to discriminate max-stable models is the CLIC (Composite Likelihood Information Criterion (Padoan et al., 2010))

$$
\mathrm{CLIC}=-2\left\{I(\hat{\beta})-\operatorname{tr}\left(\hat{J} \hat{H}^{-1}\right)\right\}
$$

with $\hat{\beta}$ as the vector which maximizes the composite likelihood $/$ in (10), tr denoting the matrix trace, $\hat{H}$ and $\hat{J}$ the Hessian and Jacobian information matrices defined by

$$
\hat{H}=-\sum_{n=1}^{N} \sum_{i=1}^{K-1} \sum_{j=i+1}^{K} \frac{\partial^{2} \log f\left(z_{n, i}, z_{n, j} ; C_{\lambda}(n), C_{\kappa}(n), \hat{\beta}\right)}{\partial \beta \partial \beta^{t}}
$$

and

$$
\hat{\jmath}=\sum_{n=1}^{N}\left\{\sum_{i=1}^{K-1} \sum_{j=i+1}^{K} \frac{\partial \log f\left(z_{n, i}, z_{n, j} ; C_{\lambda}(n), C_{\kappa}(n), \hat{\beta}\right)}{\partial \beta}\right\}\left\{\sum_{i=1}^{K-1} \sum_{j=i+1}^{K} \frac{\partial \log f\left(z_{n, i}, z_{n, j} ; C_{\lambda}(n), C_{\kappa}(n), \hat{\beta}\right)}{\partial \beta}\right\}^{\prime} .
$$

Similar to that for Akaike Information Criterion (Akaike, 1974; Burnham \& Anderson, 2004), among a set of competing models, the best one is the one with the lowest CLIC. As in Blanchet and Davison (2011) and in Nicolet et al. (2017), we rescaled CLIC by dividing by $K-1$.

\subsubsection{Confidence Interval}

A 95\% confidence interval is estimated for the range of extremal dependence $h_{0}(\lambda, \kappa)$ by the delta method (Cox, 1998), propagating the standard error on $\hat{\beta}=(\hat{\lambda}, \hat{\kappa})$ in (7). Hence, the $95 \%$ confidence interval for $h_{0}(\beta)$ is given by

$$
\left[h_{0}(\hat{\beta}) \pm \frac{\Phi^{-1}(0.975)}{\sqrt{N}} \sqrt{\nabla h_{0}(\hat{\beta})^{T} \Sigma(\hat{\beta}) \nabla h_{0}(\hat{\beta})}\right]
$$

with $\nabla h_{0}(\hat{\beta})$ the gradient of $h_{0}$ with respect to $\hat{\beta}$ and $\Sigma(\hat{\beta})$ the variance-covariance matrix of $\beta$.

\subsubsection{Alternative Model Fitting}

A disadvantage in the maximization of the composite log-likelihood / defined in (10) is that the winter maxima are taken into account for each winter separately while the covariates are considered through their 17-year moving averages (Figure 3). Thus, annual variability remains in snow depth maxima while it is smoothed in the covariates. To solve this issue, we propose to associate moving window-based and likelihood maximization-based approaches.

Let $w_{q}$ (from $w_{1}=1962-1978$ to $w_{Q}=2004-2020$, allowing to go beyond the recording period by considering "NA" values) be the 17-year moving window used in the computation of $C_{\lambda}(q)$ and $C_{\kappa}(q)$. We note $I_{q}$ as the composite log-likelihood using the standardized covariates $C_{\lambda}(q)$ and $C_{\kappa}(q)$ computed on $w_{q}$ :

$$
I_{q}(\beta)=\sum_{r \in w_{q}} \sum_{i=1}^{K-1} \sum_{j=i+1}^{K} \log f\left(z_{r, i}, z_{r, j} ; C_{\lambda}(q), C_{\kappa}(q), \beta\right) .
$$


The quantity to maximize is the sum over $q$ of $I_{q}(\beta)$ :

$$
\tilde{I}(\beta)=\sum_{q=1}^{N} I_{q}(\beta) .
$$

The quantity $\tilde{l}(\beta)$ is referred to as the alternative composite likelihood. The set of parameters to estimate remains identical to that described in section 3.2.1.

\section{Workflow}

\subsection{Standardization to Unit Fréchet}

First, winter maxima at each location are transformed into unit Fréchet variables. A GEV distribution is estimated for each station by likelihood maximization, giving estimates $(\hat{\mu}(x), \hat{\sigma}(x), \hat{\xi}(x))$ of the GEV parameters at each station location $x$. These pointwise estimates are used to transform at each position $x$ the GEV distributed snow depth maxima into a unit Fréchet distributed variable using the transformation (2).

\subsection{Stationary Case}

With the later aim of comparing the stationary and nonstationary models, we first fit the Brown-Resnick process to snow depth maxima under the hypothesis of temporal stationarity. Then we compute the extremal function stemming from the fitted process. In parallel, we estimate the pairwise extremal coefficients through the estimator introduced in Naveau et al. (2009), which is based on the madogram (Cooley et al., 2006). Whereas some classical estimators may have some difficulties to estimate the upper tail dependence of extremes (Serinaldi et al., 2015), this estimator has shown suitable performances in Bel et al. (2008) and Naveau et al. (2009). Then, we compute the class averages of these estimates in order to check the suitability of the fitted stationary Brown-Resnick process.

To compare the 3-D modified distances from one model to another, we use the anisotropy parameters $\hat{\psi}, \hat{R}_{1}$, and $\hat{R}_{2}$ estimated in the stationary case for sections 4.3,4.4, and 4.5 ("hat" is the classical notation for a parameter estimate resulting in our case from the composite likelihood maximization). Holding these parameters fixed rather than reestimating them in the nonstationary case using each covariate barely affects the results and eases much the comparison of the results.

\subsection{Moving Time Window}

A preliminary investigation of the temporal evolution of the extremal dependence is done through a data-based approach similar to the one used in Nicolet et al. (2016). This approach will allow us to motivate the interest in modeling trends in the parameters of the spatial dependence structure. In addition, it will provide results that may be compared to the results obtained by the nonstationary Brown-Resnick processes with the aim to show their consistency.

We assess the temporal evolution of the extremal dependence by fitting the stationary Brown-Resnick process on a 17-year moving window from 1970-1986 to 2000-2016 (the large number of maxima at the end of the study period allows us to go beyond 2012, the decrease in goodness of fit of max-stable processes between 17 and 13 years of observations had been rather limited regarding another data set with comparable number of stations (Nicolet et al., 2017)). We keep the same anisotropy transformation for each estimation window: we apply for all moving windows the parameters $\hat{\psi}, \hat{R}_{1}$, and $\hat{R}_{2}$ estimated in the stationary case using all the data, and we reestimate the parameters $\hat{\lambda}(q)$ and $\hat{\kappa}(q)$ for each estimation window $w_{q}$. Then, for each estimation window, we obtain the Brown-Resnick extremal function (6) and the range of extremal dependence (7).

\subsection{Nonstationary Case}

In section 4.3, we investigated the existence of temporal nonstationarity in the spatial dependence structure of snowfall maxima. The objective of the current section is to model this temporal nonstationarity by using time-dependent covariates for the two parameters $\lambda$ and $\kappa$. The Brown-Resnick process is fitted by maximizing the composite likelihood (10) using covariates $C_{\lambda}(n)=\left\{C_{\lambda, k}(n)\right\}_{k \in\left\{1, \ldots, M_{1}\right\}}$ and $C_{K}(n)=\left\{C_{K, k}(n)\right\}_{k \in\left\{1, \ldots, M_{2}\right\}}$ so as to estimate the functions $\lambda(n)=\lambda_{0}+\Sigma_{m=1}^{M_{1}} C_{\lambda, m}(n) \lambda_{m}$ and $\kappa(n)=\kappa_{0}+\Sigma_{m=1}^{M_{2}} C_{\kappa, m}(n) \kappa_{m}$. Again, we held the anisotropy parameters $\hat{\psi}, \hat{R}_{1}$, and $\hat{R}_{2}$ fixed to the values estimated in the stationary case of section 4.2. Models with one and two covariates are fitted, using the covariates of Table 1 but excluding the pairs with a correlation larger than 0.8 in absolute value. We consider two cases: the first one with one covariate for $\lambda$ and/or $\kappa$, and the second one with two covariates. Concerning the models with one covariate, we considered 


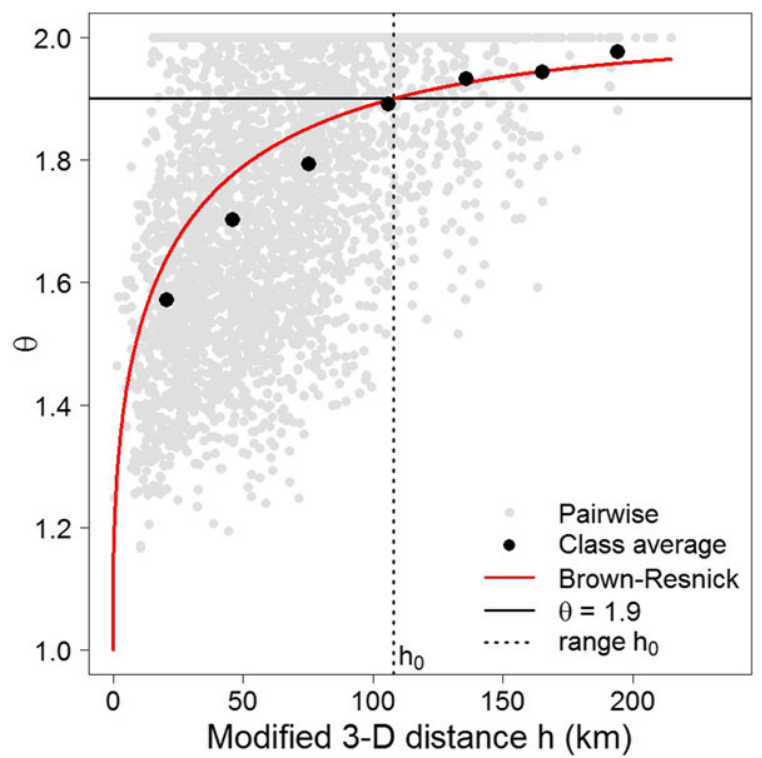

Figure 5. Stationary case (section 5.1). Estimated extremal function under the hypothesis of temporal stationarity (red curve). Grey dots represent the madogram-based pairwise estimations (Naveau et al., 2009) of the extremal coefficient for every pair of stations, and black dots represent the distance class averages (seven distance classes of equal length are defined from $0 \mathrm{~km}$ to the maximum pairwise distance $215 \mathrm{~km}$ ). The range of extremal dependence (equation (7) is $\hat{h}_{0}=108 \mathrm{~km}$. three possibilities:

1. a trend in $\lambda$ with $\kappa$ stationary (i.e., $\lambda(n)=\lambda_{0}+\lambda_{1} C_{\lambda}(n)$ and $\left.\kappa(n)=\kappa_{0}\right)$;

2. a trend in $\kappa$ with $\lambda$ stationary (i.e., $\kappa(n)=\kappa_{0}+\kappa_{1} C_{\kappa}(n)$ and $\lambda(n)=\lambda_{0}$ ); and

3. a trend in both $\lambda$ and $\kappa$ with the same covariate.

In the case of the models with two covariates, we tried all the possibilities for each considered pair of covariates: $\left(M_{1}, M_{2}\right)=$ $(2,0),(0,2),(1,1),(1,2),(2,1)$ and $(2,2)$. The selection of the best model is done through CLIC.

\subsection{Alternative Composite Likelihood}

Finally, the model selected by CLIC in section 4.4 is fitted by maximizing the alternative composite log-likelihood $I$ defined in (16), in order to compare the two approaches and to assess the influence of the annual variability of maxima on the results.

\section{Results}

\subsection{Stationary Case}

The estimated anisotropy parameters are $\hat{\psi}=51.52^{\circ}$ (standard deviation: $\left.6.90^{\circ}\right), \hat{R}_{1}=1.79(0.35)$, and $\hat{R}_{2}=36.66(7.38)$, and the estimated scale and shape parameters are $\hat{\lambda}=10.0$ (1.03) and $\hat{\kappa}=0.71$ (0.055), respectively. The extremal function stemming from this estimated process is close to the class averages of the pairwise estimates (Figure 5) showing the quality of the estimated Brown-Resnick process. These estimated parameters $\hat{\psi}, \hat{R}$, and $\hat{R}_{2}$ for anisotropy are used for all the models considered in the following. In order to fairly compare stationary and nonstationary models, the stationary model is reestimated by keeping the anisotropy parameters fixed and the considered CLIC is the one computed on this latter model. With this model, the range of extremal dependence is $\hat{h}_{0}=108 \mathrm{~km}$ (standard deviation: $25 \mathrm{~km}$ ).

\subsection{Time Moving Window}

Figure 6a shows the temporal evolution of the extremal function considering 17-year estimation windows and holding the anisotropy parameters fixed to the values obtained in section 5.1. We observe a positive temporal trend for the extremal function at large distance and therefore a negative temporal trend for the extremal dependence. This is confirmed by Figure $6 \mathrm{~b}$, which highlights a strong negative temporal trend in the range of extremal dependence. While the first estimates have to be interpreted carefully due to the low number of observations, we can observe from 1985 to 1992 a division by 2 of the range of extremal dependence (from $200 \mathrm{~km}$ to $100 \mathrm{~km}$, which corresponds to the range estimated in the stationary case), and a stabilization from 1992 to the end of the study period. Although the first confidence intervals are extremely wide, the estimates at the beginning of the decrease (for instance, more than $200 \mathrm{~km}$ in 1985) lie outside the last confidence intervals which extend from about 50 to $150 \mathrm{~km}$ in 1992, so the trend in extremal dependence is significant.

The corresponding estimates of $\lambda$ and $\kappa$ show strongly positive trends (Figures $6 c$ and $6 \mathrm{~d}$ ), which confirms the interest in allowing temporal trends in these parameters.

\subsection{Nonstationary Brown-Resnick Model}

As mentioned before, we keep the anisotropy parameters $\psi, R_{1}$, and $R_{2}$ fixed by using the estimates of the stationary case because this eases the comparison of the results and barely affects the results. For instance, Figure 7 shows a comparison between the parameters $\lambda_{0}, \lambda_{1}, \kappa_{0}$, and $\kappa_{1}$ obtained when anisotropy is held fixed or when anisotropy is estimated together with these parameters. The observed differences concerning the estimates of $\lambda_{0}$ are due to the fact that this parameter is strongly related to the anisotropy parameters and particularly to $R_{1}$. The estimation of this parameter is thus more sensitive when it is estimated together with anisotropy. The estimates of the three other parameters are very similar with points very close to the diagonal.

According to CLIC, apart from three covariates, the models with one covariate are better when a trend is modeled in the two parameters $\lambda$ and $\kappa$ (Figure 8). The covariates NAO and minimum temperature at $1,800 \mathrm{~m}$ show a better CLIC when a trend is modeled in the parameter $\lambda$ only, while the best model using snow precipitation ratio at $1,800 \mathrm{~m}$ models just a trend in $\kappa$. 
(a)

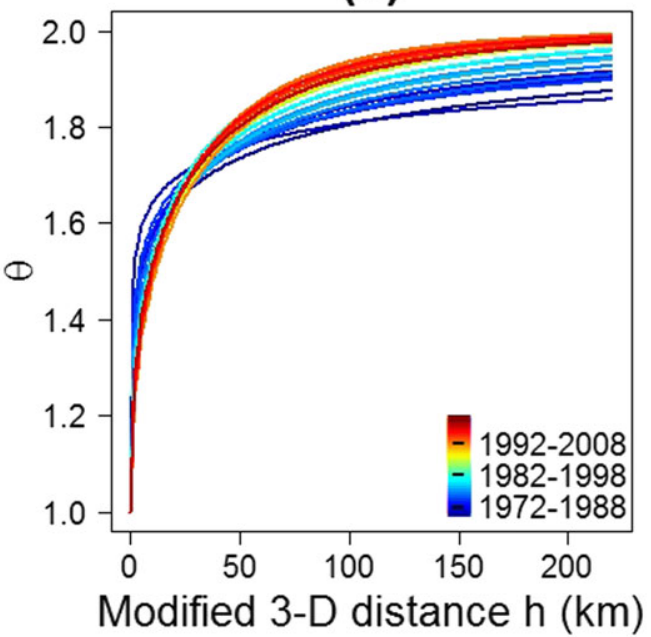

(c)

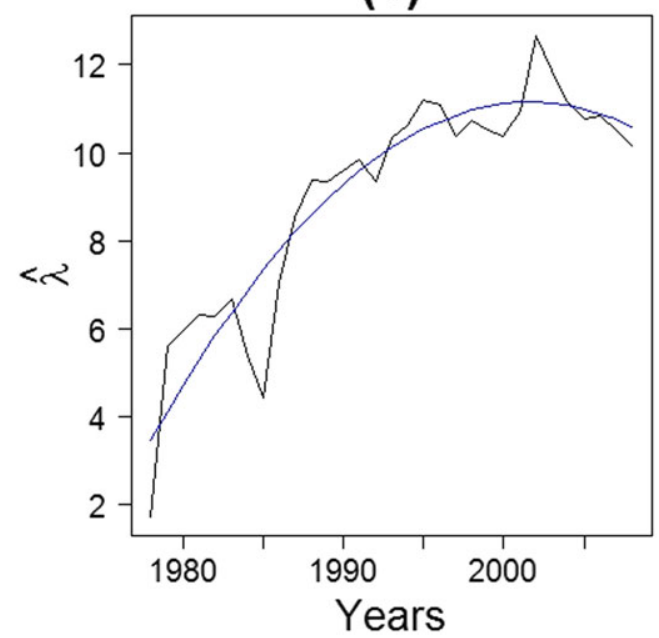

(b)

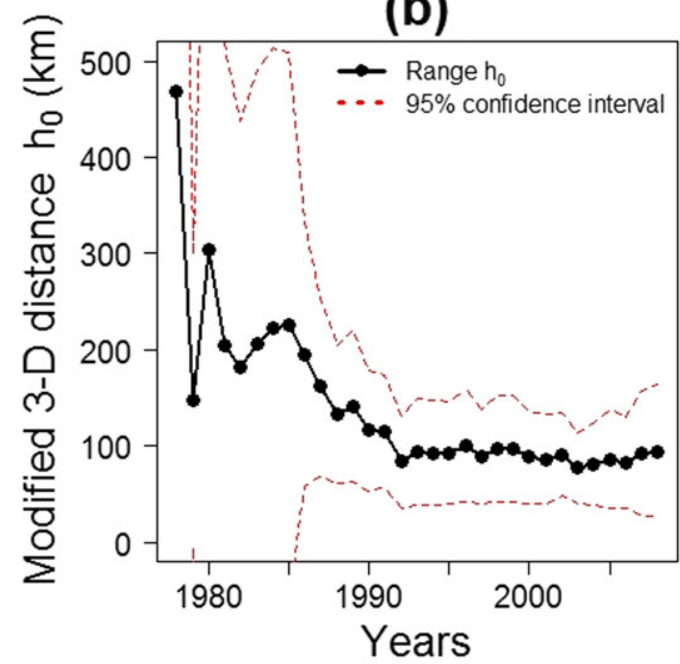

(d)

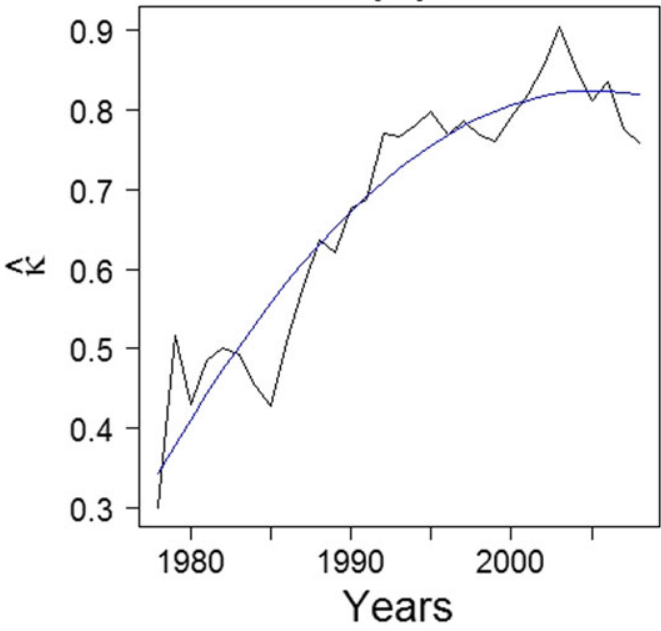

Figure 6. Time moving window (section 5.2). (a) Temporal evolution of the fitted extremal functions, from oldest time windows (blue curves) to the most recent ones (red curves). (b) Temporal evolution of the range of extremal dependence. The range is expressed as a function of the 3-D modified distance. It is plotted (black dots) as a function of the center of the considered estimation window. The associated $95 \%$ confidence interval is evaluated by the delta method (equation (14). (c, d) Temporal evolution of the estimates of (a) $\lambda$ and (b) $\kappa$. The $X$ axis represents the center of the 17-year time window. The blue curves show for each parameter a second degree polynomial trend fitted by least squares.

The best model with one covariate according to the CLIC uses snow precipitation ratio at 1,800 $\mathrm{m}$ as covariate for $\kappa$ and no trend in $\lambda$. The second, third, and fourth ones are those using cumulated snowfall at 1,800 $\mathrm{m}$, snow precipitation ratio at $1,800 \mathrm{~m}$, and cumulated snowfall at $2,400 \mathrm{~m}$ as covariate for both parameters $\lambda$ and $\kappa$, respectively. The other covariates outperforming the stationary model are mean temperature and maximum temperature at both elevations considered. Other models with one covariate are not better than the stationary model.

In order to see if the covariates are positively or negatively correlated to the extremal dependence of extreme snow depths, we determine the best model according to the CLIC for each covariate. We use the parameters of these best models to assess the sign of the contribution of each covariate on $\theta(100)$, the extremal function measured at the modified 3-D distance $100 \mathrm{~km}$. This distance is close to the range of extremal dependence estimated in the stationary case. The contribution of a covariate to $\theta(100)$ is referred to as positive (respectively, negative) when $\theta(100)$ increases (respectively, decreases) with respect to an increase in the covariate. Since larger $\theta$ means less dependence at extreme level, a positive contribution to $\theta(100)$ means a 

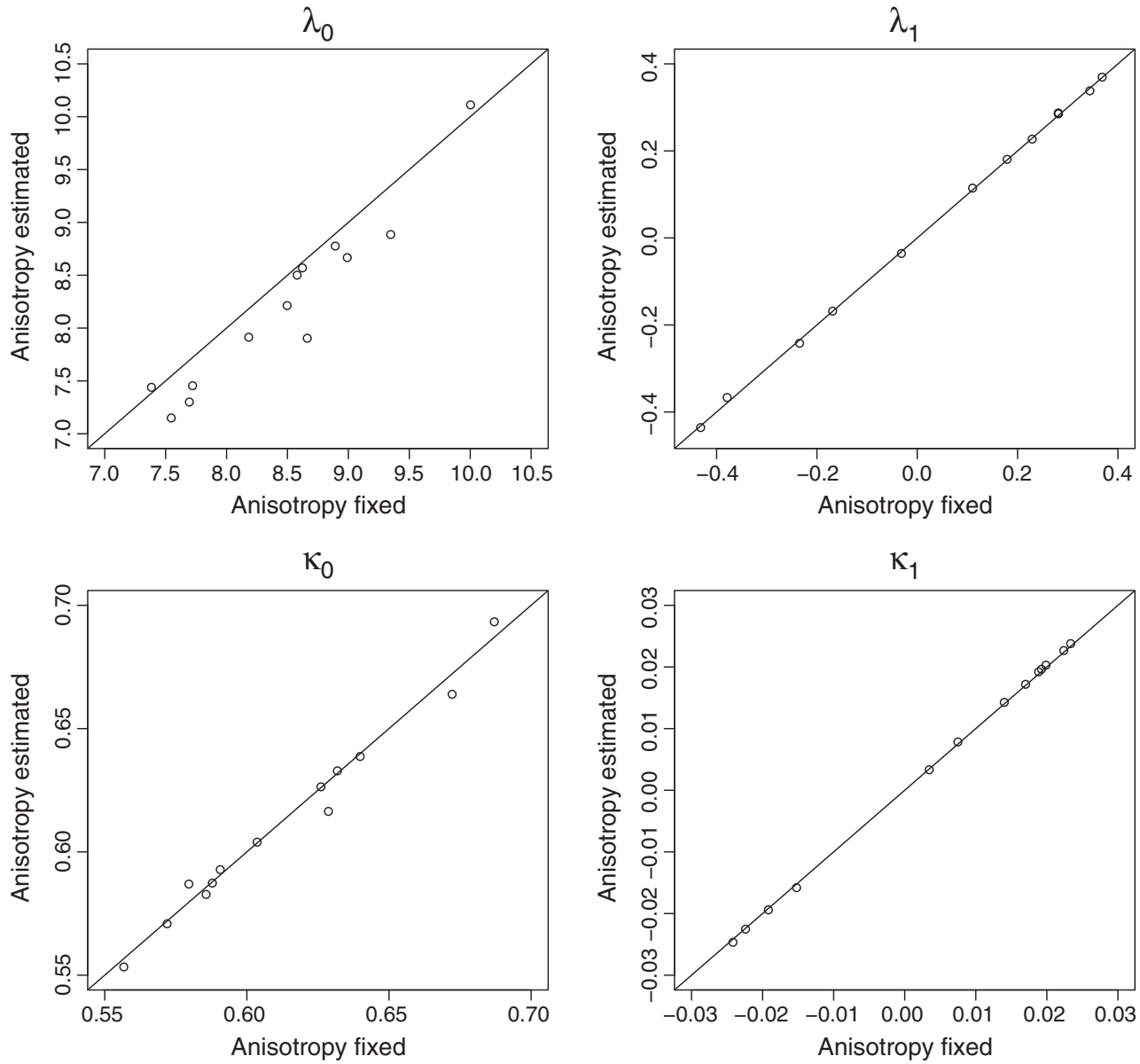

Figure 7. Nonstationary Brown-Resnick model (section 5.3). For each parameter $\lambda_{0}$ (top left), $\lambda_{1}$ (top right), $\kappa_{0}$ (bottom left), and $\kappa_{1}$ (bottom right): comparison between the estimates obtained with fixed anisotropy ( $x$ axis) and those obtained when they are estimated together with anisotropy parameters ( $y$ axis). Each point represents one model with a unique covariate modeling a trend in both $\lambda$ and $\kappa$.

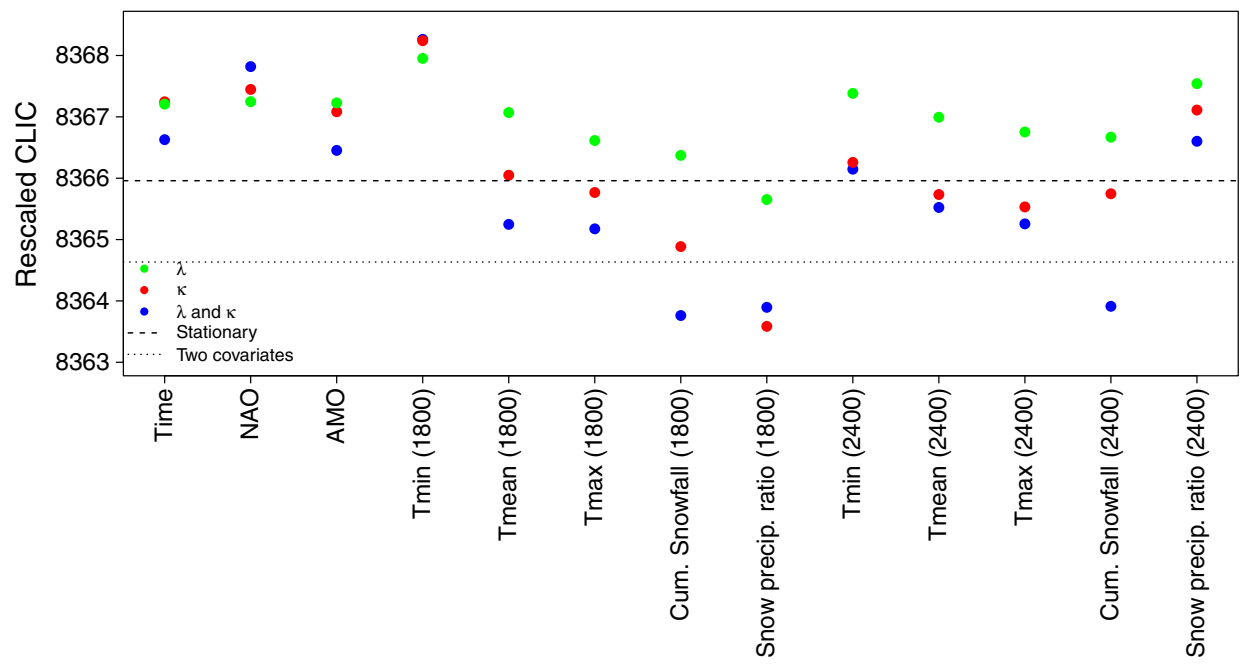

Figure 8. Nonstationary Brown-Resnick model (section 5.3): rescaled CLIC for the models using one covariate. The covariates are time ("Year"), NAO, AMO, minimum temperature, mean temperature, maximum temperature, cumulated snowfall, and snow precipitation ratio. The dashed and dotted lines represent the CLIC for the stationary model (estimated by fixing the anisotropy parameters) and for the best model with two covariates (snow precipitation ratio at $1,800 \mathrm{~m}$ and minimum temperature at $2,400 \mathrm{~m}$ as covariates for $\kappa$ with $\lambda$ constant), respectively. 
Table 2

Nonstationary Brown-Resnick Model (Section 5.3): Models With One Covariate

\begin{tabular}{lrrccl}
\hline Covariates & $\hat{\lambda}_{0}$ & $\hat{\lambda}_{1}$ & $\hat{\kappa}_{0}$ & $\hat{\kappa}_{1}$ & Contribution \\
\hline Tmean (1800) & 7.5 & 3.4 & 0.56 & 0.22 & positive \\
Tmax (1800) & 7.7 & 3.7 & 0.57 & 0.23 & positive \\
Cumulated snowfall (1800) & 7.7 & -3.8 & 0.59 & -0.22 & negative \\
Snow precip. ratio (1800) & 10.1 & - & 0.62 & -0.014 & negative \\
Tmean (2400) & 8.5 & 2.3 & 0.60 & 0.17 & positive \\
Tmax (2400) & 8.2 & 2.8 & 0.59 & 0.20 & positive \\
Cumulated snowfall (2400) & 7.4 & -4.3 & 0.58 & -0.24 & negative \\
\hline
\end{tabular}

Note. Estimated parameters and sign of the contribution to the extremal coefficient $\theta(100)$. Only the models outperforming the stationary model are considered. $\hat{\lambda}_{0}, \hat{\lambda}_{1}, \hat{\kappa}_{0}$, and $\hat{\kappa}_{1}$ denote the estimated parameters for each model. No estimate for $\hat{\lambda}_{1}$ means that the best model with the considered covariate is the one with the trend in $\kappa$ only. The column "Contribution" indicates if the extremal coefficient at the distance $100 \mathrm{~km}$ (i.e., $\theta(100)$ ), arbitrarily chosen, increases (positive) or decreases (negative) with respect to an increase in the covariate. Note that a positive contribution to $\theta(100)$ means a decrease in the extremal dependence at the distance $100 \mathrm{~km}$.

negative contribution of the covariate to the extremal dependence at $100 \mathrm{~km}$. This definition is consistent in practice because the function $\theta(100)$ of the covariate is always monotonic in the observed range of this covariate (i.e., between the minimum and the maximum measured values). Moreover, the results are also consistent whether trends are considered in lambda only, kappa only, or both variables (not shown). The covariates showing a positive contribution to $\theta(100)$ are mean daily temperature and maximum daily temperature (Table 2 ). This means that these covariates have a negative contribution to the extremal dependence of snow depths. On the contrary, cumulated snowfall and snow precipitation ratio have a positive contribution to the extremal dependence of snow depths. The other covariates (time, NAO, AMO, minimum daily temperature) do not outperform the stationary model and have no significant contribution.

None of the models using two covariates shows a better CLIC than the best model with one covariate (Figure 8). The model using snow precipitation ratio at $1,800 \mathrm{~m}$ as unique covariate for the smoothness parameter $\lambda$ is thus the best one according to CLIC. With this model, consistently with the results obtained using time moving window (section 5.2), the extremal coefficient $\theta(h)$ increases with time at large distances, which implies a negative trend in the extremal dependence (Figure 9a). As using time moving window, the estimated range of extremal dependence shows a strong decrease during the 1980s and then a relative consistency after 1993 (Figure 9b). The estimates of the range of extremal dependence is much larger during the first part of the study period (sometimes larger than $800 \mathrm{~km}$ ). However, again, these estimates must be considered with caution due to the lack of observations before 1983. The evolution of the extremal function with respect to the snow precipitation ratio at $1,800 \mathrm{~m}$ (Figure $9 \mathrm{c}$ ) confirms that the extremal dependence tends to increase when the snow precipitation ratio increases (i.e., $\theta(100)$ decreases).

\subsection{Alternative Composite Likelihood}

The temporal evolution of the extremal function and the range of extremal dependence stemming from the model using snow precipitation ratio at $1,800 \mathrm{~m}$ as unique covariate for $\lambda$ but fitted by maximizing the alternative composite likelihood $\tilde{I}$ defined in (16) is similar as in the case of the classical composite likelihood (Figures 10a and 10b). However, this temporal evolution is much smoother when using the alternative composite likelihood $\tilde{I}$. The range of extremal dependence spans a much narrower interval with values not larger than $205 \mathrm{~km}$ (3-D modified distance), which is consistent with the empirical case (section 5.2) if we discard the first three estimation windows for which few observations are available. The extremal function as function of snow precipitation ratio at $1,800 \mathrm{~m}$ shows as before an increase in extremal dependence (i.e., decrease in $\theta(100)$ ) when snow precipitation ratio increases but with much less variability (Figure 10c).

\section{Discussion}

\subsection{Anisotropy in the Spatial Dependence and Comparison With Extreme Snowfalls}

The results obtained for snow depths under the stationary hypothesis in section 5.1 can be compared to those provided by Nicolet et al. (2017) who fitted a stationary Brown-Resnick process to snowfall maxima in the French Alps by maximum composite likelihood in the context of model. To the best of our knowledge, this is 
(a)

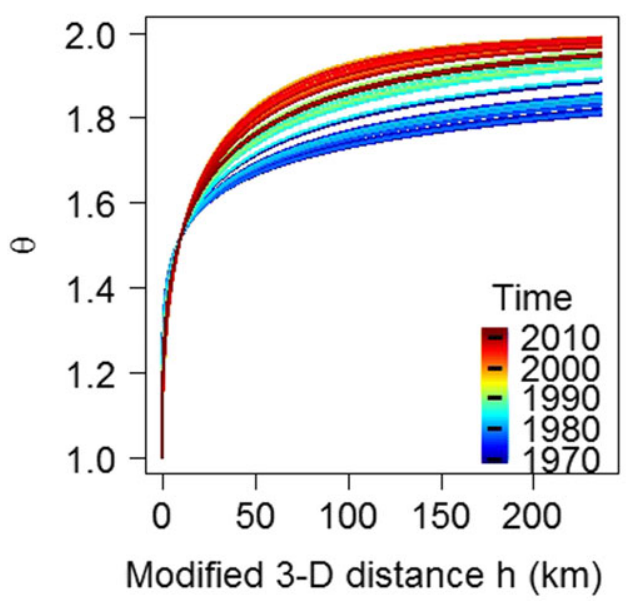

(b)

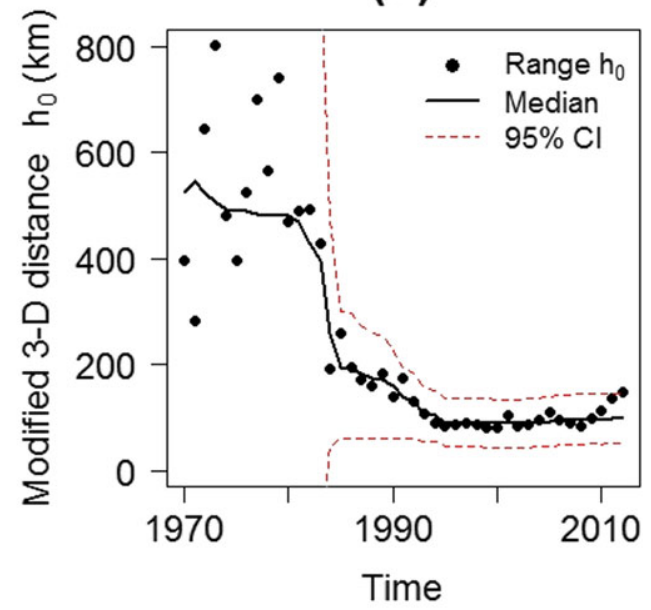

(c)

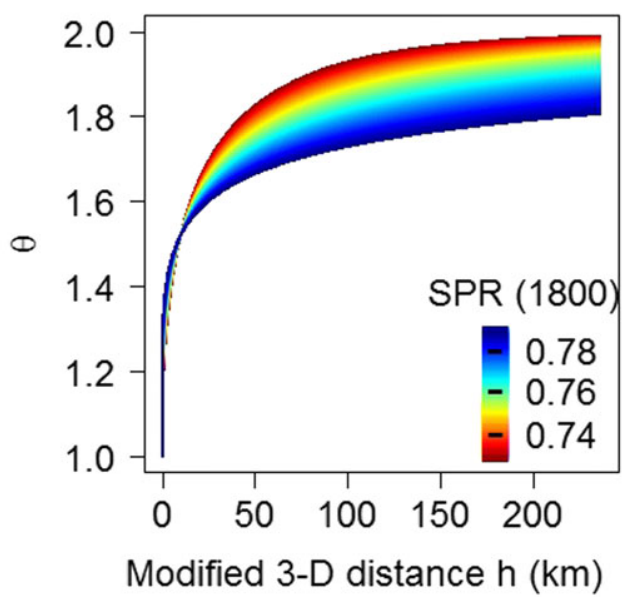

Figure 9. Nonstationary Brown-Resnick model (section 5.3). Model selected by CLIC with snow precipitation ratio at $1,800 \mathrm{~m}$ as covariate for the smoothness parameter $\kappa$. (a) Temporal evolution of the extremal function stemming from the model. (b) Temporal evolution of range of extremal dependence. The solid line represents the median range of extremal dependence computed on a 17 -year moving window. The associated $95 \%$ confidence interval is evaluated by the delta method. (c) Evolution of the extremal function as function of snow precipitation ratio at 1,800 m (from 0.73 to 0.79 ).

the first time that the spatial dependence in extremes of these two snow-related variables can be compared over the same region.

For both variables, the estimated anisotropy angle ( $\hat{\psi}=51.52^{\circ}$ for snow depths and $\hat{\psi}=37.28^{\circ}$ for snowfalls $)$ corresponds to the orientation of the main massifs and valleys in the French Alps. This pattern has also been observed for extreme snow depths in Switzerland (Blanchet \& Davison, 2011) and for extreme precipitation in the Appalachians (Padoan et al., 2010) and in the French Mediterranean region (Blanchet \& Creutin, 2017), which confirms its robustness. Also, recently, Blanchet et al. (2018) highlighted the impact of the evolution of the atmospheric system on the direction and the strength of the anisotropy of extreme precipitation in West Africa. This anisotropy may be interpreted as the effect of orography on atmospheric fluxes generating extreme precipitations. One can note though that anisotropy is less marked for extreme snow depths than for extreme snowfalls (with $\hat{R}_{1}=1.79$ against 3.22 for extreme snowfalls), and the effect of elevation is also slightly weaker $\left(\hat{R}_{2}=36.66\right.$ against 39.95$)$, which may arise from the fact that the interaction with atmospheric flows is weaker in the case of snow on the ground than in the case of snowfalls. 
(a)

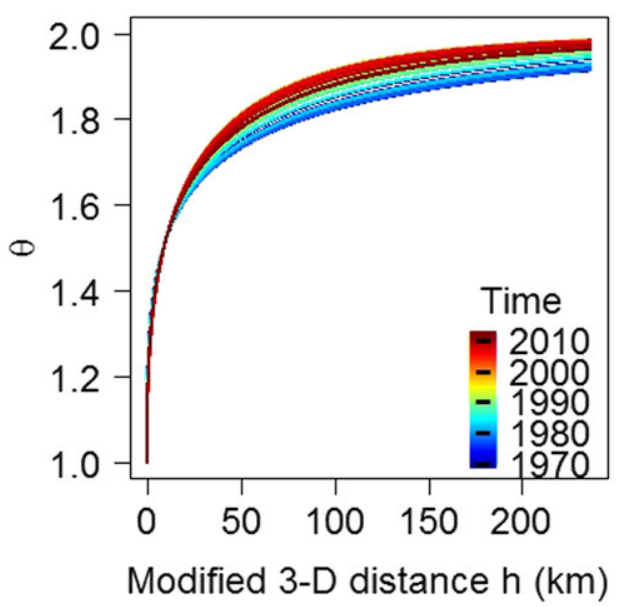

(b)

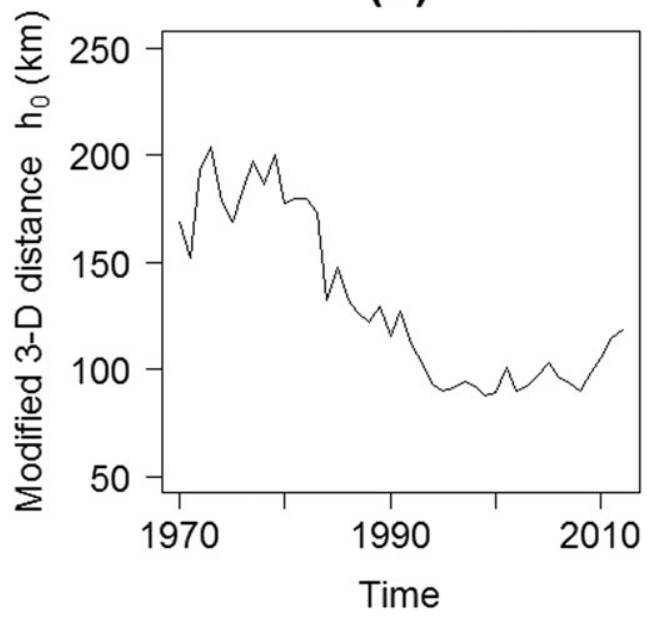

(c)

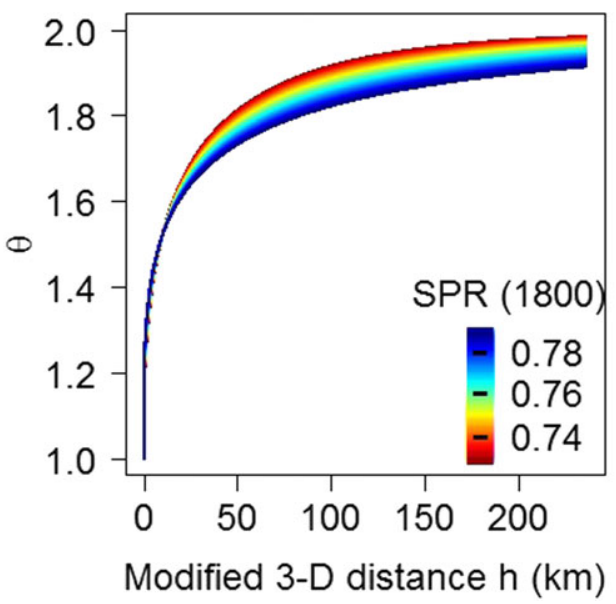

Figure 10. Alternative composite likelihood (section 5.4). Model with snow precipitation ratio at $1,800 \mathrm{~m}$ as covariate for the smoothness parameter $\kappa$ maximizing the alternative composite likelihood $I$. (a) Temporal evolution of the extremal function stemming from the model. (b) Temporal evolution of the range of extremal dependence. (c) Evolution of the extremal function as function of snow precipitation ratio at $1,800 \mathrm{~m}$ (from 0.73 to 0.79 ).

Comparing snow depth and snowfall extremal coefficients shows that snow depth maxima are more spatially dependent than snowfall maxima for a large majority of pairs of stations (Figure 11). As interpreted in Gaume et al. (2013), this is probably due to cumulative effects involved in the formation of snow cover which strongly smooths the evolution of extreme snow depths with space.

\subsection{Temporal Changes}

The decreasing trend in the extremal dependence of snow depths in the French Alps is similar to that found in Nicolet et al. (2016) for snowfalls in the same area. These two variables both show a strong decrease in the range of extremal dependence concentrated during the 1980s, and then a relative consistency after 1990. This is the first time that such an effect is inferred on extreme snow depths. The consistency with extreme snowfalls over the same area pleads for its robustness and its geophysical meaning. As already discussed (Figure 1), it was not necessarily expected due to the strong differences in the two variables. This decrease is concomitant with the period of strongest decrease in cumulated snowfall and snow precipitation ratio, and with the period of strongest increase in temperature and AMO (Figure 3).

One may wonder about a possible influence of the stationarity hypothesis in the marginal distributions on the nonstationarity observed in the spatial dependence structure, with a potential transfer of a temporal trend in the margins to the dependence structure. Indeed, among the 82 stations of the data set, we found 21 


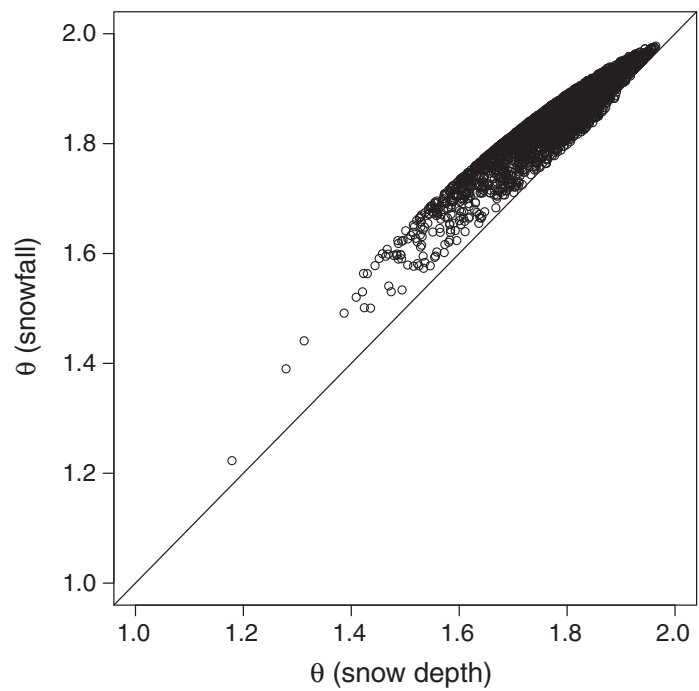

Figure 11. Comparison between snow depth extremal coefficients (this study) and snowfall extremal coefficients (Nicolet et al., 2017) for all the pairs of stations. stations with a significant negative temporal trend in the location GEV parameter $\mu$ and three with a positive trend according to a likelihood ratio test. We further fitted the Brown-Resnick process with snow precipitation ratio at $1,800 \mathrm{~m}$ as covariate of $\kappa$ to the unit Fréchet data set computed by taking into account the temporal trends for these 24 stations, which provided very similar results (Appendix B). Hence, the decreasing trend in the spatial dependence of snow depth maxima is not artificially created by the stationary hypothesis in the margins.

One may also question the issue of data availability and the impact of the changes in the station network on the results. The procedure was reproduced on a reduced data set of 13 stations, chosen in order to have a quite constant spatial coverage during the study period. Despite the larger uncertainty due to small size of the sample, we found very similar results (Appendix C).

An additional issue concerning the methodology is the length of the moving window which was set as 17 years for this study. Several values were tried to smooth the covariates, and we kept the one which optimized the CLIC. Other lengths like 15 or 19 years produced very similar results. For the sake of simplicity, the same length of 17 years was used for the moving time window (section 4.3) and for the alternative composite likelihood (section 4.5).

\subsection{Climate Control}

The most relevant covariates to model trends in the spatial dependence structure of extreme snow depths are those related to precipitation and temperature: mean temperature, maximum temperature, cumulated snowfall, and snow precipitation ratio at $1,800 \mathrm{~m}$. However, the use of minimum temperature or snow precipitation ratio at $2,400 \mathrm{~m}$ leads to models less suitable than the stationary model. The inefficiency of snow precipitation ratio at $2,400 \mathrm{~m}$ can be explained by the fact that at this elevation level, the snow precipitation ratio is always close to 1 during the entire study period (Figure 3) making its explicative power very small.

The covariates mean temperature and maximum temperature have a negative contribution to the extremal dependence of snow depths in the French Alps, while cumulated snowfall and snow precipitation ratio have a positive contribution. This is consistent with the results of Nicolet et al. (2016) concerning extreme snowfalls in the French Alps.

Hence, the decreasing temporal trend in the spatial dependence of extreme snow depths seems to be due at first to a decrease of the snow precipitation ratio caused by the increase of temperature over the study period, and particularly in the context of the 1980s climate regime shift (Reid et al., 2016). Specifically, temperature increase makes snowfalls more isolated in space. Indeed, when temperature is moderately cold, only the highest stations experience snow while rain falls at low elevations (Nicolet et al., 2016). This leads to spatially less smooth snow depth variations and less coherent patterns for snow depth maxima.

As in the case of snowfall maxima, we cannot exclude a magnitude effect with stronger dependence in extreme snow depths during snowier winters. Indeed, even if snow precipitation ratio at 2,400 $\mathrm{m}$ is one of the less efficient covariates (due to a very low effect of snow/rain partitioning at this elevation), cumulated snowfall at 2,400 $\mathrm{m}$ is one of the most relevant covariates. Consequently, the decrease in cumulated snowfall observable during the winter season since the 1980s (Figure 3) seems an additional cause for the decrease in the spatial dependence of extreme snow depths.

Contributions of AMO and NAO to extremal dependence of snow depth maxima, although having a positive sense (not indicated in Table 2) consistent with the correlations computed in Nicolet et al. (2016) for snowfall maxima, are nonsignificant. This nonsignificance can be explained by the fact that many phenomena are involved between synoptic patterns and snow on the ground.

\subsection{Pros and Cons of the Proposed Modeling Approach}

Theoretical and applied studies dealing with temporal aspects within the spatial dependence structure of max-stable processes (Davis et al., 2013a, 2013b; Embrechts et al., 2016; Huser \& Davison, 2014; Raillard et al., 2014) model the short-range temporal dependence of extremes and do not consider a possible long-range 
temporal evolution of the dependence structure. This paper lays a first stone regarding the modeling of temporal trends at climate time scale in the spatial dependence of extremes, which is an important point for managing the issues related to spatial extremes and anticipating their evolution under climate change. Although the approach introduced in this paper is quite simple, with linear trends in the parameters of the spatial dependence structure, the results are consistent with Nicolet et al. (2016) who applied a data-based approach to snowfall maxima. We showed that the annual variability in snow depth maxima can be smoothed by using the alternative composite likelihood $I$. Reducing the annual variability in snow depth maxima has the effect to smooth the temporal evolution of their spatial dependence, making it easier to interpret. In addition, the robustness of our results is granted by the step-by-step approach we propose: from empirical estimations on moving time windows to a full model taking into account long range patterns in data and covariates coupled with a rigorous model selection procedure.

The major improvement of the model-based approach introduced in this paper compared to the approaches developed in Nicolet et al. (2016) and in Blanchet et al. (2018) is the explicit incorporation of suitable covariates into the modeling. The use of moving windows, as done in Nicolet et al. (2016), Blanchet et al. (2018), and in section 4.2 is a flexible way for detecting temporal trends in spatial dependence because it does not require any hypothesis on the nature of the trend (e.g., linear or polynomial of order 2; or more). However, it is not usable for anticipating the future evolution of the spatial dependence. On the contrary, the use of covariates has the advantage that it may allow anticipating the evolution of the extremal dependence of snow extremes. Indeed, the various future climate change scenarios now increasingly available (e.g., Verfaillie et al., 2017, 2018) for the French Alps) could be easily used with the best fitted model.

A limitation of the considered model is the difficulty to efficiently combine more than one covariate. Indeed, we did not consider joint models involving two of the most relevant covariates (cumulated snowfall, mean temperature, maximum temperature, and snow precipitation ratio at 1,800 $\mathrm{m}$ ) because these covariates are strongly correlated. The best joint models show a compensation effect between the two covariates, showing the complication to use them. None of the models with two covariates not too much correlated is able to outperform the best model with one covariate, showing that all the considered combinations of covariates are not relevant enough.

Finally, considering nonlinear trends in the spatial dependence structure may improve the prediction performance.

\section{Conclusion and Outlooks}

In this paper we introduce a way to account for the temporal nonstationarity in extremes, which we apply to study the evolution of the spatial dependence in extreme snow depths in the French Alps since 1970. Several climate covariates are considered to model trends in the spatial structure of dependence and the best model is selected by CLIC. We find a strong negative temporal trend in the spatial dependence of extreme snow depths with a strong decrease in the range of extremal dependence during the 1980s. The most relevant covariates to explain these temporal patterns are cumulated snowfall, snow precipitation ratio (considered at 1,800 $\mathrm{m}$ above sea level where snow-rain partitioning changed), and temperature (maximum and average). Hence, the decrease in extremal dependence seems mainly due to the effect of the increase in temperature on the snow precipitation ratio at low elevation and to a decrease in the winter cumulated snowfall. These results are consistent with those obtained for extreme snowfalls in the same region in previous studies, showing that the spatial dependence of these two snow variables are similarly impacted by climate change.

The space-time approach introduced in this article, which permits to model the spatial dependence of extremes as function of appropriate time-dependent covariates, may be very useful for quantifying spatial extremes and managing the related risk and its evolution under ongoing climate change. It may provide insights on climate drivers and open the door to predict spatial extremes under climate change scenarios. This approach is here applied to extreme snow depths, but it may be fruitful for many other geophysical variables.

\section{Appendix A: Covariates}

In addition to time, we use several covariates which summarize the winter climate of the French Alps over the study period. The French Alps are divided into 23 massifs (see Figure 2a), which are generally assumed to be homogeneous in terms of meteorological conditions for a given elevation. In each massif, the meteorological 
(a)

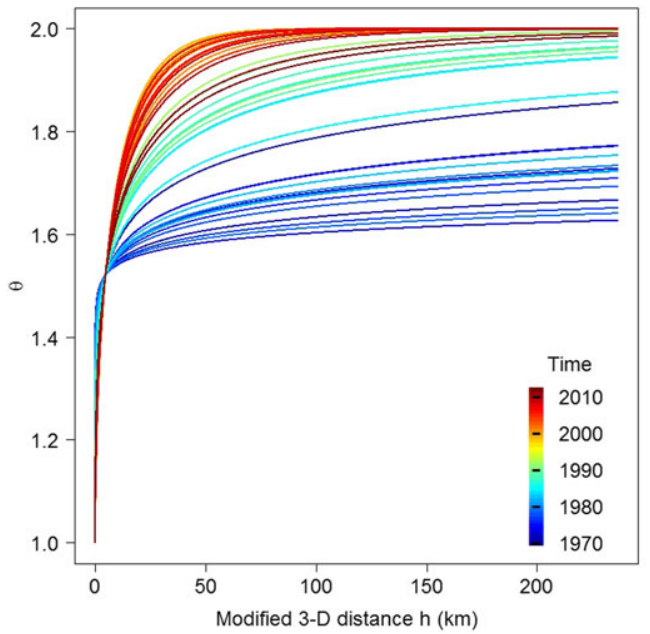

(b)

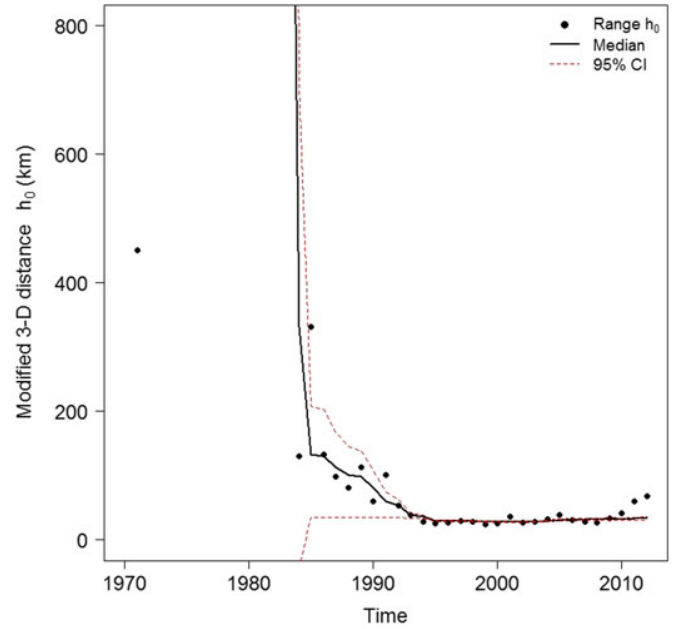

Figure B1. Same as Figures 9a and 9b), but taking into account the temporal trends in the parameter $\mu$ for the 24 stations for which this trend is significant.
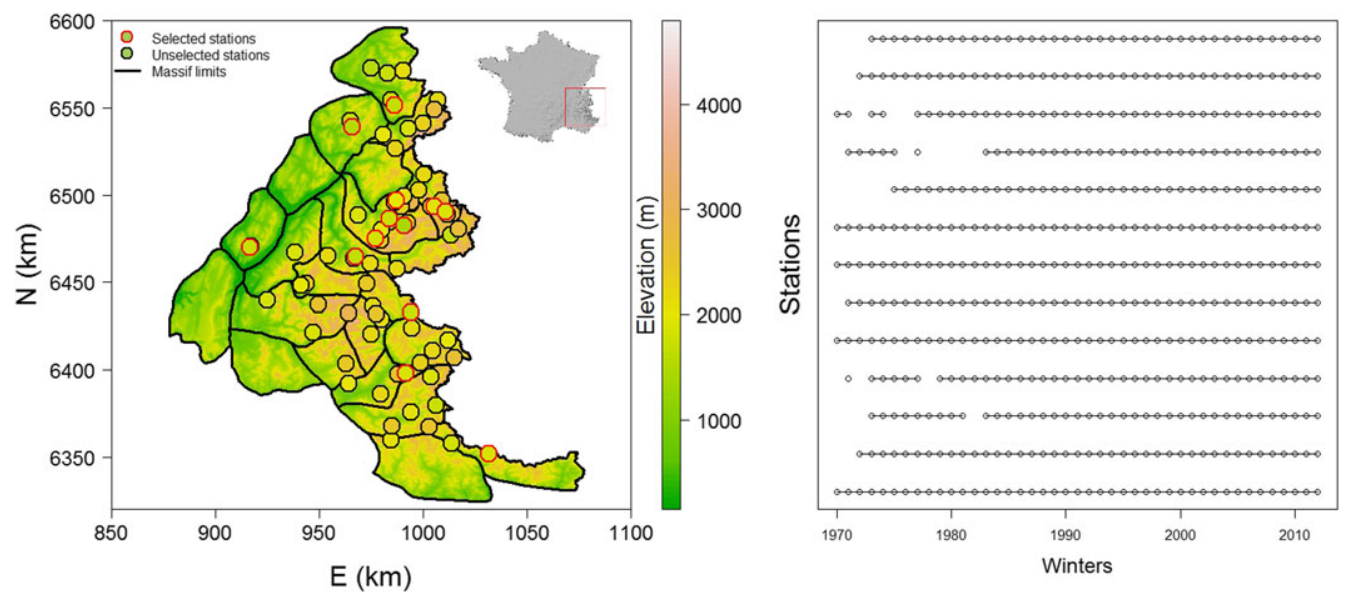

Figure C1. (a) Location of the 13 selected stations. (b) Data availability for the 13 selected stations.

(a)

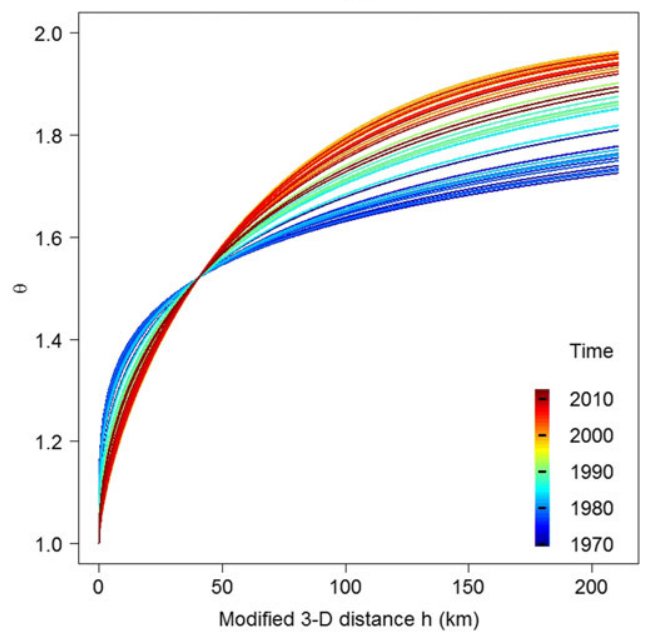

(b)

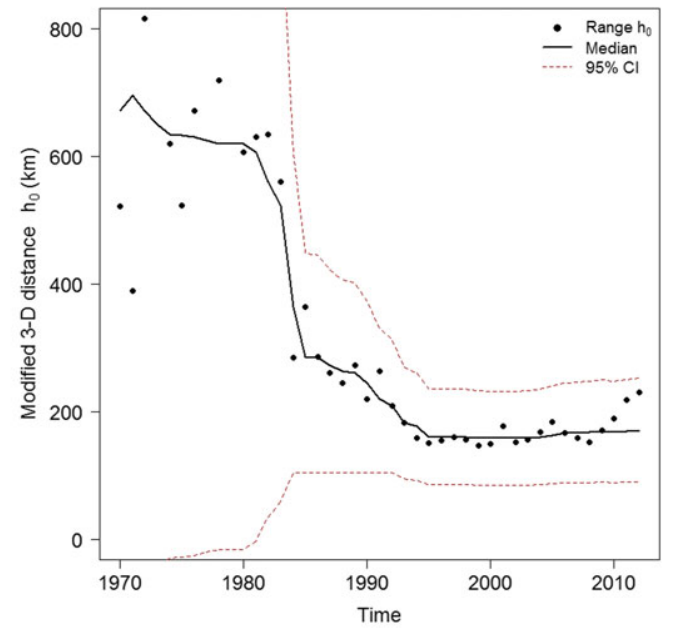

Figure C2. Same as Figures $9 \mathrm{a}$ and $9 \mathrm{~b}$ with a reduced data set of 13 stations. 
conditions (temperature and precipitation) are available all over the study period as a function of elevation through reanalyses (Durand, Laternser, et al., 2009) provided by the SAFRAN (Système d'Analyse Fournissant des Renseignements Atmosphérique à la Neige) model (Durand et al., 1993; Durand et al., 1999). From these reanalyses, the cumulated snowfall, snow precipitation ratio (cumulated snow precipitation divided by total — snow and rain — precipitation), and the daily maximum, mean, and minimum temperature are calculated for two elevation levels $(1,800 \mathrm{~m}$ and 2,400 $\mathrm{m}$ ) for each winter and for each massif. Then, the mean of the 23 massif values is computed for each winter in order to have, for each variable and each winter, a single value for the entire French Alps, notwithstanding the large variability of mean annual conditions (Durand, Laternser, et al., 2009). We do not consider reanalyses of winter mean snow water equivalent or winter mean snow depth (Durand, Giraud, et al., 2009; Vionnet et al., 2012) as covariates because they are too close from the variable of interest.

We also consider NAO (Jones et al., 1997; Osborn, 2006) and AMO (Enfield et al., 2001; Kaplan et al., 1998) indices through winter anomalies evaluated from November to April over the study period. NAO and AMO variables summarize the predominant oscillating patterns in the winter climate of the French Alps, in terms of pressure/precipitation and temperature, respectively. Rather than the more commonly used detrended version of AMO (Enfield et al., 2001), we use here the nondetrended version which includes the recent climate warming signal in addition to oscillating patterns (Kaplan et al., 1998). In the western Alps, a negative NAO anomaly is associated with colder temperatures and more intense snowfall (López-Moreno et al., 2011).

\section{Appendix B: Impact of the Nonstationarity of the Marginal Distributions}

Applying likelihood ratio tests for the GEV parameter $\mu$ reveal that 24 stations exhibit a significant temporal trend, including 21 negative trends and 3 positive trends. We fitted the Brown-Resnick process to the unit Fréchet data set obtained by taking into account the trends in the $\mu$ parameter of these 24 stations. Figure B1 shows that the first estimates of the range of extremal dependence are extremely large, due to the flatness of the extremal function. However, one can still observe a negative temporal trend in the 1980s. Thus, the negative temporal trend observed when fitting a Brown-Resnick process with nonstationnary spatial dependence structure (section 5.3) cannot be attributed to a possible transfer of a temporal trend in the marginal distribution to the spatial dependence structure.

\section{Appendix C: Impact of the Network Evolution}

In order to check the influence of the evolution of the station network on the results, we fitted the Brown-Resnick process to a reduced data set of 13 stations with a fair spatial coverage (Figure C1a), chosen in order to have a quite constant spatial coverage during the study period (Figure C1b). Despite the larger uncertainty due to smaller sample size, one can still observe the temporal trend (Figure C2).

Acknowledgments This work has been supported by a grant from LabEx OSUG@2020 (Investissements d'avenir-ANR10 LABX56). We thank the hundreds of snow observers and the Météo-France staff involved in the collection and constitution of the data sets used in this study. Meteorological data used in this publication are available through the Public Data portal of Météo-France available at https://donneespubliques. meteofrance.fr/ and are free of use for research purpose. Irstea, IGE, and CNRM are members of LabEx OSUG@2020.

\section{References}

Akaike, H. (1974). A new look at the statistical model identification. Automatic Control, IEEE Transactions on, 19(6), 716-723. Asadi, P., Davison, A. C., \& Engelke, S. (2015). Extremes on river networks. arXiv preprint arXiv:1501.02663.

Bel, L., Bacro, J. N., \& Lantuéjoul, C. (2008). Assessing extremal dependence of environmental spatial fields. Environmetrics, $19,163-182$. https://doi.org/10.1002/env.863

Beniston, M., Farinotti, D., Stoffel, M., Andreassen, L. M., Coppola, E., Eckert, N., et al. (2018). The European mountain cryosphere: A review of its current state, trends, and future challenges. The Cryosphere, 12(2), 759-794.

Blanchet, J., Aly, C., Vischel, T., Panthou, G., Sané, Y., \& Kane, M. D. (2018). Trend in the co-occurrence of extreme daily rainfall in West Africa since 1950. Journal of Geophysical Research: Atmospheres, 123, 1536-1551. https://doi.org/10.1002/2017JD027219

Blanchet, J., \& Creutin, J.-D. (2017). Co-occurrence of extreme daily rainfall in the French Mediterranean region. Water Resources Research, 53, 9330-9349. https://doi.org/10.1002/2017WR020717

Blanchet, J., \& Davison, A. C. (2011). Spatial modeling of extreme snow depth. Annals of Applied Statistics, 5(3), $1699-1725$. https://doi.org/10.1214/11-AOAS464

Brown, B., \& Resnick, S. I. (1977). Extreme values of independent stochastic processes. Journal of Applied Probability, 14, $732-739$.

Burnham, K. P., \& Anderson, D. R. (2004). Multimodel inference: Understanding AIC and BIC in model selection. Sociological methods \& research, 33(2), 261-304.

Castruccio, S., Huser, R., \& Genton, M. G. (2016). High-order composite likelihood inference for max-stable distributions and processes. Journal of Computational and Graphical Statistics, 25(4), 1212-1229.

Coles, S. (2001). An introduction to statistical modeling of extreme values. London: Springer.

Cooley, D., Cisewski, J., Erhardt, R. J., Jeon, S., Mannshardt, E., Omolo, B. O., \& Sun, Y. (2012). A survey of spatial extremes: Measuring spatial dependence and modeling spatial effects. Revstat, 10(1), 135-165.

Cooley, D., Naveau, P., \& Poncet, P. (2006). Variograms for spatial max-stable random fields. In P. Bertail, P. Soulier, \& P. Doukhan (Eds.), Dependence in probability and statistics (Vol. 187, pp. 373-390). New York: Springer. 
Cox, C. (1998). Delta method. In P. Armitage \& T. Colton (Eds.), Encyclopedia of biostatistics (Vol. 2, pp. 1125-1127). Chichester: John Wiley \& Sons.

Davis, R. A., Klüppelberg, C., \& Steinkohl, C. (2013a). Max-stable processes for modeling extremes observed in space and time. Journal of the Korean Statistical Society, 42(3), 399-414. https://doi.org/10.1016/j.jkss.2013.01.002

Davis, R. A., Klüppelberg, C., \& Steinkohl, C. (2013b). Statistical inference for max-stable processes in space and time. Journal of the Royal Statistical Society: Series B (Statistical Methodology), 75(5), 791-819.

Davison, A. C., Padoan, S. A., \& Ribatet, M. (2012). Statistical modeling of spatial extremes. Statistical Science, 27(2), $161-186$.

de Haan, L. (1984). A spectral representation for max-stable processes. Annals of Probability, 12(4), 1194-1204.

Dombry, C., Éyi-Minko, F., \& Ribatet, M. (2012). Conditional simulation of max-stable processes. Biometrika, 100(1), $111-124$.

Durand, Y., Brun, E., Mérindol, L., Guyomarc'h, G., Lesaffre, B., \& Martin, E. (1993). A meteorological estimation of relevant parameters for snow models. Annals of Glaciology, 18, 65-71.

Durand, Y., Giraud, G., Brun, E., Mérindol, L., \& Martin, E. (1999). A computer-based system simulating snowpack structures as a tool for regional avalanche forecasting. Journal of Glaciology, 45(151), 469-485.

Durand, Y., Giraud, G., Laternser, M., Etchevers, P., Mérindol, L., \& Lesaffre, B. (2009). Reanalysis of 47 years of climate in the French Alps (1958-2005): Climatology and trends for snow cover. Journal of Applied Meteorology and Climatology, 48(12), $2487-2512$.

Durand, Y., Laternser, M., Giraud, G., Etchevers, P., Lesaffre, B., \& Mérindol, L. (2009). Reanalysis of 44 yr of climate in the French Alps (1958-2002): Methodology, model validation, climatology, and trends for air temperature and precipitation. Journal of Applied Meteorology and Climatology, 48(3), 429-449.

Ehrler, C., Seidel, K., \& Martinec, J. (1997). Advanced analysis of snow cover based on satellite remote sensing for the assessment of water resources. IAHS Publications-Series of Proceedings and Reports-Intern Assoc Hydrological Sciences, 242, 93-102.

Embrechts, P., Koch, E., \& Robert, C. (2016). Space-time max-stable models with spectral separability. Advances in Applied Probability, 48(A), 77-97.

Enfield, D., Mestas-Nunez, A., \& Trimble, P. (2001). The Atlantic multidecadal oscillation and its relation to rainfall and river flows in the continental U.S. Geophysical Research Letters, 28(10), 2077-2080.

Gaume, J., Chambon, G., Eckert, N., \& Naaim, M. (2012). Relative influence of mechanical and meteorological factors on avalanche release depth distributions: An application to French Alps. Geophysical Research Letters, 39, L12401. https://doi.org/10.1029/2012GL051917

Gaume, J., Eckert, N., Chambon, G., Naaim, M., \& Bel, L. (2013). Mapping extreme snowfalls in the French Alps using max-stable processes. Water Resources Research, 49, 1079-1098. https://doi.org/10.1002/wrcr.20083

Geis, J., Strobel, K., \& Liel, A. (2011). Snow-induced building failures. Journal of Performance of Constructed Facilities, $26(4), 377-388$.

Huser, R., \& Davison, A. C. (2014). Space-time modelling of extreme events. Journal of the Royal Statistical Society, Series B, 76(2), 439-461. https://doi.org/10.1111/rssb.12035

Jones, P. D., Jonsson, T., \& Wheeler, D. (1997). Extension to the North Atlantic Oscillation using early instrumental pressure observations from Gibraltar and South-West Iceland. International Journal of Climatology, 17(13), 1433-1450.

Kabluchko, Z., Schlather, M., \& de Haan, L. (2009). Stationary max-stable fields associated to negative definite functions. Annals of Probability, 37(5), 2042-2065.

Kaplan, A., Cane, M., Kushnir, Y., Clement, A., Blumenthal, M., \& Rajagopalan, B. (1998). Analyses of global sea surface temperature 1856-1991. Journal of Geophysical Research, 103(18), 18,567-18,589.

Keller, F., Goyette, S., \& Beniston, M. (2005). Sensitivity analysis of snow cover to climate change scenarios and their impact on plant habitats in alpine terrain. Climatic Change, 72(3), 299-319.

Koenig, U., \& Abegg, B. (1997). Impacts of climate change on winter tourism in the Swiss Alps. Journal of sustainable tourism, 5(1), 46-58.

Leadbetter, M. (1983). Extremes and local dependence in stationary sequences. Zeit. Wahrscheinl.-theorie, 65, 291-306.

López-Moreno, J. I., Vicente-Serrano, S. M., Morán-Tejeda, E., Lorenzo-Lacruz, J., Kenawy, A., \& Beniston, M. (2011). Effects of the North Atlantic Oscillation (NAO) on combined temperature and precipitation winter modes in the Mediterranean mountains: Observed relationships and projections for the 21st century. Global and Planetary Change, 77(1), 62-76.

Naveau, P., Guillou, A., Cooley, D., \& Diebolt, J. (2009). Modelling pairwise dependence of maxima in space. Biometrika, 96(1), $1-17$.

Nicolet, G., Eckert, N., Morin, S., \& Blanchet, J. (2016). Decreasing spatial dependence in extreme snowfall in the French Alps since 1958 under climate change. Journal of Geophysical Research: Atmospheres, 121, 8297-8310. https://doi.org/10.1002/2016JD025427

Nicolet, G., Eckert, N., Morin, S., \& Blanchet, J. (2017). A multi-criteria leave-two-out cross-validation procedure for max-stable process selection. Spatial Statistics, 22, 107-128.

Oesting, M., Bel, L., \& Lantuéjoul, C. (2017). Sampling from a max-stable process conditional on a homogeneous functional with an application for downscaling climate data. Scandinavian Journal of Statistics, 45, 382-404.

Osborn, T. J. (2006). Recent variations in the winter North Atlantic Oscillation. Weather, 61(12), 353-355.

Padoan, S. A., Ribatet, M., \& Sisson, S. A. (2010). Likelihood-based inference for max-stable processes. Journal of the American Statistical Association, 105(489), 263-277.

Raillard, N. (2011). Modélisation du comportement extrême de processus spatio-temporels. applications en océanographie et météorologie (Ph.D. thesis), Université, Rennes 1.

Raillard, N., Ailliot, P., \& Yao, J. (2014). Modeling extreme values of processes observed at irregular time steps: Application to significant wave height. Annals of Applied Statistics, 8(1), 622-647. https://doi.org/10.1214/13-AOAS711

Reich, B. J., \& Shaby, B. A. (2012). A hierarchical max-stable spatial model for extreme precipitation. Annals of Applied Statistics, 6(4), 1430.

Reid, P. C., Hari, R. E., Beaugrand, G., Livingstone, D. M., Marty, C., Straile, D., et al. (2016). Global impacts of the 1980s regime shift. Global Change Biology, 22, 682-703. https://doi.org/10.1111/gcb.13106

Ribatet, M. (2013). Spatial extremes: Max-stable processes at work. J. Soc. Fr. Stat., 154(2), 156-177.

Schlather, M., \& Tawn, J. A. (2003). A dependence measure for multivariate and spatial extreme values: Properties and inference. Biometrika, 90(1), 139-156.

Schweizer, J., Mitterer, C., \& Stoffel, L. (2009). On forecasting large and infrequent snow avalanches. Cold Regions Science and Technology, 59(2-3), 234-241.

Serinaldi, F., Bárdossy, A., \& Kilsby, C. G. (2015). Upper tail dependence in rainfall extremes: Would we know it if we saw it? Stochastic Environmental Research and Risk Assessment, 29(4), 1211-1233. https://doi.org/10.1007/s00477-014-0946-8

Shang, H., Yan, J., \& Zhang, X. (2011). El Niño-Southern Oscillation influence on winter maximum daily precipitation in California in a spatial model. Water Resources Research, 47, 11. https://doi.org/10.1029/2011WR010415

Steinkohl, C. K. (2013). Statistical modelling of extremes in space and time using max-stable processes (PhD thesis), Technische Universität München.

Taylor, D. A. (1979). A survey of snow loads on the roofs of arena-type buildings in Canada. Canadian Journal of Civil Engineering, 6(1), 85-96. 
Thibaud, E., Mutzner, R., \& Davison, A. (2013). Threshold modeling of extreme spatial rainfall. Water Resources Research, 49, 4633-4644. Verfaillie, D., Déqué, M., Morin, S., \& Lafaysse, M. (2017). The method ADAMONT v1. 0 for statistical adjustment of climate projections applicable to energy balance land surface models. Geoscientific Model Development, 10(11), 4257.

Verfaillie, D., Lafaysse, M., Déqué, M., Eckert, N., Lejeune, Y., \& Morin, S. (2018). Multi-component ensembles of future meteorological and natural snow conditions for $1500 \mathrm{~m}$ altitude in the Chartreuse mountain range, Northern French Alps. The Cryosphere, $12(4), 1249$.

Vionnet, V., Brun, E., Morin, S., Boone, A., Faroux, S., Le Moigne, P., et al. (2012). The detailed snowpack scheme Crocus and its implementation in SURFEX v7.2. Geoscientific Model Development, 5, 773-791.

Westra, S., \& Sisson, S. A. (2011). Detection of non-stationarity in precipitation extremes using a max-stable process model. Journal of Hydrology, 406(1-2), 119-128.

Wipf, S., Stoeckli, V., \& Bebi, P. (2009). Winter climate change in alpine tundra: Plant responses to changes in snow depth and snowmelt timing. Climatic change, 94(1-2), 105-121. 